\title{
Mechanistic Insights into the Chiral Phosphoramide-Catalyzed, Enantioselective Crossed-Aldol Reactions of Aldehydes
}

\author{
Scott E. Denmark* and Tommy Bui \\ Roger Adams Laboratory, Department of Chemistry, University of Illinois, 600 South Mathews \\ Avenue, Urbana, Illinois 61801

\section{SUPPORTING INFORMATION}

\section{Table of Contents}

General Experimental

Hammett Studies

Natural Abundance ${ }^{12} \mathrm{C} /{ }^{13} \mathrm{C}$ Isotope Effects

${ }^{12} \mathrm{C} /{ }^{13} \mathrm{C}$ Isotope Effects for $\mathbf{2 d}$

${ }^{12} \mathrm{C} /{ }^{13} \mathrm{C}$ Isotope Effects for $\mathbf{2 f}$

Arrhenius Activation Parameters

Kinetic Data for 2d

Kinetic Data for $\mathbf{2 f}$

References
Page

$S 1-S 2$

S12-S16

S12-S14

S15-S16

S17-S41

S17-S32

S32-S40

$S 41$

\section{General Experimental}

All reactions were performed in oven-dried $\left(140^{\circ} \mathrm{C}\right)$ or flame-dried glassware under an atmosphere of dry argon or nitrogen. The following reaction solvents were distilled from the indicated drying agents: dichloromethane $\left(\mathrm{P}_{2} \mathrm{O}_{5}\right)$, chloroform $\left(\mathrm{P}_{2} \mathrm{O}_{5}\right)$, tetrahydrofuran (sodium, benzophenone), solvents for crystallization benzene (Fisher ACS grade), hexanes (Fisher ACS grade) were used as received. Solvents for chromatography and filtration were technical grade and distilled from the indicated drying agents: hexane $\left(\mathrm{CaCl}_{2}\right)$; ethyl acetate $\left(\mathrm{K}_{2} \mathrm{CO}_{3}\right)$; 
dichloromethane $\left(\mathrm{CaCl}_{2}\right)$; isopropanol (Fisher ACS grade) was used as received. Column chromatography was performed using EM Science 230-400-mesh silica gel. Anisaldehyde, 4tolualdehyde, benzaldehyde, and 4-(trifluoromethyl)benzaldehyde were freshly distilled before use. All reaction temperatures correspond to internal temperatures measured by Teflon-coated thermocouples unless otherwise noted.

${ }^{1} \mathrm{H}$ NMR, ${ }^{13} \mathrm{C}$ NMR spectra were recorded on $400 \mathrm{MHz},{ }^{1} \mathrm{H}\left(100 \mathrm{MHz},{ }^{13} \mathrm{C}\right), 500 \mathrm{MHz},{ }^{1} \mathrm{H}$ $\left(126 \mathrm{MHz},{ }^{13} \mathrm{C}\right)$ spectrometers. Spectra were referenced to residual chloroform $\left(\mathrm{d} 7.26 \mathrm{ppm},{ }^{1} \mathrm{H}\right.$; d $\left.77.00 \mathrm{ppm},{ }^{13} \mathrm{C}\right)$. Chemical shifts are reported in ppm (d); multiplicities are indicated by $\mathrm{s}$ (singlet), d (doublet), t (triplet), q (quartet), quint (pentet), m (multiplet), and br (broad). Coupling constants, $J$, are reported in Hertz. All ${ }^{1} \mathrm{H}$ and ${ }^{13} \mathrm{C}$ NMR assignments are corroborated by 2D experiments (HETCOR and COSY). Spectra available on request from denmark@scs.uiuc.edu.

Low-resolution electron impact (EI) mass spectra were obtained with a typical ionization voltage of $70 \mathrm{eV}$. Chemical ionization (CI) spectra were obtained using methane as the carrier gas. Data are reported in the form of $(\mathrm{m} / \mathrm{z})$. Infrared spectra (IR) are reported in $\mathrm{cm}^{-1}$ with indicated relative intensities: $\mathrm{s}$ (strong, 67-100\%); m (medium, 34-66\%); w (weak, 0-33\%). Melting points (mp) were determined in sealed tubes and are corrected. Bulb-to-bulb distillations were performed in a Kugelrohr, and boiling points (bp) correspond to the uncorrected air bath temperatures (ABT). Analytical thin-layer chromatography was performed on Merck silica gel plates with QF-254 indicator. Visualization was accomplished with 2,4dinitrophenylhydrazine or $\mathrm{KMnO}_{4}$.

Optical rotation data was obtained on a digital polarimeter and are reported as follows: concentration ( $c=\mathrm{g} / 100 \mathrm{~mL}$ ), and solvent. Analytical supercritical fluid chromatography (SFC) was performed on packed-column SFC with built-in photometric detector $(\lambda=220,258 \mathrm{~nm})$ using Daicel Chiralpak OD, AD, and AS columns. Retention times $\left(t_{\mathrm{R}}\right)$ and peak areas for SFC were obtained from reporting integrators.

Enolate $\mathbf{1}^{1}$ and catalyst $(R, R)-\mathbf{3}^{2}$ were prepared as previously described. The catalyst is also commercially available from Obiterresearch, (waboulanger@obiterresearch.com). 


\section{Experimental Procedures}

\section{Hammett Studies}

\section{General Procedure I}

\section{(R)-(2,2-Dimethoxy-1,1-dimethylethyl)benzenemethanol (4a)}
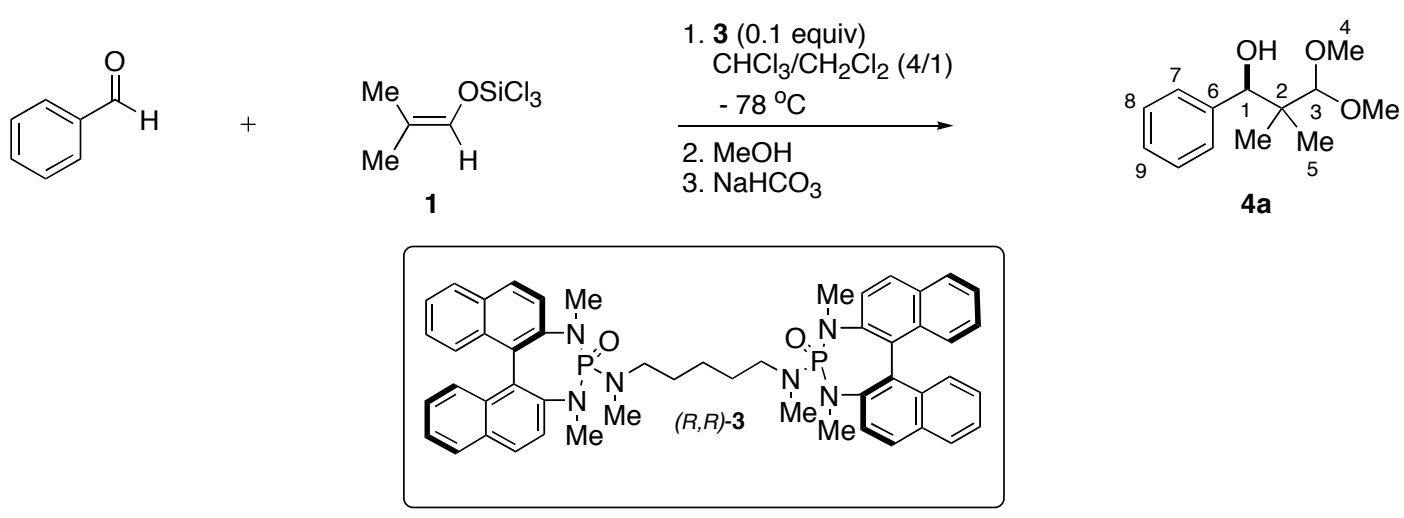

To a stirred solution of $3(84.3 \mathrm{mg}, \quad 0.1 \mathrm{mmol}, 0.1$ equiv) in $4 \mathrm{~mL}$ of chloroform/methylene chloride (4/1) in a 2-necked, round-bottomed flask fitted with a nitrogen inlet adapter and thermocouple at $-78{ }^{\circ} \mathrm{C}$ was added enolate 1 (226.1 mg, $1.1 \mathrm{mmol}, 1.1$ equiv) dropwise via syringe. Five minutes later, freshly distilled benzaldehyde (102 $\mu \mathrm{L}, 1 \mathrm{mmol})$ was added dropwise via syringe. The reaction mixture was stirred at $-78{ }^{0} \mathrm{C}$ under $\mathrm{N}_{2}$ for $8 \mathrm{~h}$. Dry methanol $(15 \mathrm{~mL})$ was then added dropwise at $-78{ }^{\circ} \mathrm{C}$, and the reaction mixture was stirred for $45 \mathrm{~min}$. The solution was allowed to warm to room temperature (30 min) and was then quickly poured into a rapidly stirred, cold, aqueous sodium bicarbonate solution $(25 \mathrm{~mL})$. The resulting mixture was stirred at room temperature for $2 \mathrm{~h}$. A cloudy white solution was observed during this period. The mixture was filtered through Celite and the aqueous layer was separated and extracted with methylene chloride $(3 \times 25 \mathrm{~mL})$. The combined organic layers were dried over sodium sulfate, filtered, and concentrated in vacuo. Silica gel column chromatography (hexanes/ethyl acetate, 5/1) followed by bulb- to-bulb distillation afforded $192.6 \mathrm{mg}(86 \%)$ of $4 \mathbf{a}$ as a clear, colorless oil.

\section{Data for 4a}

bp: $\quad 140{ }^{\circ} \mathrm{C}(0.02 \mathrm{mmHg}, \mathrm{ABT})$

1슬 $: \quad\left(400 \mathrm{MHz}, \mathrm{CDCl}_{3}\right)$

7.23-7.33 (m, 5 H, H-Aryl), 4.76 (d, $J=2.2,1 \mathrm{H}, \mathrm{HC}(5)), 4.05$ (s, $1 \mathrm{H}$, 
$\mathrm{H}(\mathrm{C}(2)), 3.87(\mathrm{~d}, J=2.2,1 \mathrm{H}, \mathrm{OH}), 3.58\left(\mathrm{~s}, 3 \mathrm{H}, \mathrm{H}_{3} \mathrm{C}(3)\right), 3.56\left(\mathrm{~s}, 3 \mathrm{H}, \mathrm{H}_{3} \mathrm{C}(3)\right)$, $0.92\left(\mathrm{~s}, 3 \mathrm{H}, \mathrm{H}_{3} \mathrm{C}(4)\right), 0.76$ (s, $\left.3 \mathrm{H}, \mathrm{H}_{3} \mathrm{C}(4)\right)$

${ }^{13}$ C NMR: $\quad\left(400 \mathrm{MHz}, \mathrm{CDCl}_{3}\right)$

$141.13(\mathrm{C}(6)), 128.01(\mathrm{C}(7)), 127.47(\mathrm{C}(9)), 127.14(\mathrm{C}(8)), 114.02 \quad(\mathrm{C}(2)), 77.95$

$(\mathrm{C}(5)), 58.82(\mathrm{C}(3)), 58.67(\mathrm{C}(3)), 43.62(\mathrm{C}(1)), 21.13(\mathrm{C}(4)), 17.04(\mathrm{C}(4))$

IR: $\quad\left(\mathrm{CHCl}_{3}\right)$

3629 (w), 3479 (br), 3009 (s), 2942 (s), 2836 (m), 1471 (m), 1453 (m), 1390 (w), 1339 (w), 1237 (w), 1190 (m), 1104 (s), 1070 (s), 1016 (s), 955 (w), 705 (m)

MS: $\quad(\mathrm{FI})$

$224\left(\mathrm{M}^{+}, 0.4\right), 192\left(\mathrm{M}^{+}-\mathrm{MeOH}, 4\right), 106\left(\mathrm{C}_{6} \mathrm{H}_{5} \mathrm{CHO}, 33\right), 86\left(\left(\mathrm{CH}_{3}\right)_{2} \mathrm{CCHOMe}\right.$, 100)

Opt. Rot.: $\quad[\alpha]_{\mathrm{D}}^{24}-4.56(c=0.74, \mathrm{EtOH})$

TLC: $\quad R_{f} 0.30$ (hexane/EtOAc, 5/1) [silica gel, DNP]

SFC: $\quad(R)-\mathbf{4 a}, t \mathrm{R} 2.78 \min (70.2 \%) ;(S)-\mathbf{4 a}, t \mathrm{R} 3.19 \min (29.8 \%)$ (Column: OD, $\mathrm{MeOH}$ $5 \%$, pressure $150 \mathrm{psi}$, flow $3.0 \mathrm{~mL} / \mathrm{min}$ )

Analysis: $\quad \mathrm{C}_{13} \mathrm{H}_{20} \mathrm{O}_{3}(224.30)$
Calcd:
C, 69.61;
$\mathrm{H}, 8.99 \%$
Found:
C, 69.33;
$\mathrm{H}, 9.02 \%$

\section{(R)-(2,2-Dimethoxy-1,1-dimethylethyl)-4-methylphenylmethanol (4b)}
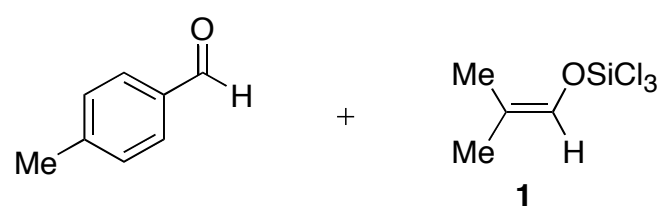
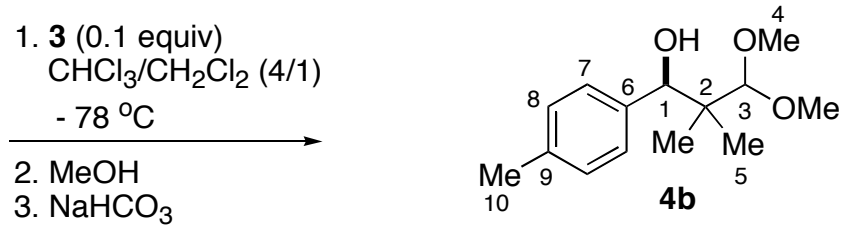

Following General Procedure I, a solution of 3 ( $84.3 \mathrm{mg}, 0.1 \mathrm{mmol}, 0.1$ equiv), trichlorosilyl enolate 1 (226.1 mg, $1.1 \mathrm{mmol}, 1.1$ equiv), and 4-methylbenzaldehyde (118 $\mu \mathrm{L}$, $1.0 \mathrm{mmol})$ in $4 \mathrm{~mL}$ of $\mathrm{CHCl}_{3} / \mathrm{CH}_{2} \mathrm{Cl}_{2}$ (4/1) was stirred at $-78{ }^{\circ} \mathrm{C}$ under nitrogen for $12 \mathrm{~h}$. Workup and purification by silica gel column chromatography using hexanes/ethyl acetate, 5/1 as eluent provided the desired aldol product, which was further purified by bulb-to-bulb distillation to afford $214 \mathrm{mg}$ (90\%) of $\mathbf{4 b}$ as clear, colorless oil. 


\section{$\underline{\text { Data for } \mathbf{4 b}}$}

bp: $\quad 160{ }^{\circ} \mathrm{C}(0.02 \mathrm{mmHg}, \mathrm{ABT})$

1ㅁN $\underline{\text { NMR: }} \quad\left(400 \mathrm{MHz}, \mathrm{CDCl}_{3}\right)$

$7.20(\mathrm{~d}, J=8.1,2 \mathrm{H}, 2 \times \mathrm{HC}(7)), 7.11(\mathrm{~d}, J=8.1,2 \mathrm{H}, 2 \times \mathrm{HC}(8)), 4.71$ (s, $1 \mathrm{H}$, $\mathrm{HC}(5)), 4.04$ (s, $1 \mathrm{H}, \mathrm{H}(\mathrm{C}(2)), 3.84$ (s, $1 \mathrm{H}, \mathrm{OH}$ ), 3.55 (s, $\left.3 \mathrm{H}, \mathrm{H}_{3} \mathrm{C}(3)\right), 3.53$ (s, 3 $\left.\mathrm{H}, \mathrm{H}_{3} \mathrm{C}(3)\right), 2.34$ (s, $\left.3 \mathrm{H}, \mathrm{H}_{3} \mathrm{C}(10)\right), 0.91$ (s, $\left.3 \mathrm{H}, \mathrm{H}_{3} \mathrm{C}(4)\right), 0.75$ (s, $\left.3 \mathrm{H}, \mathrm{H}_{3} \mathrm{C}(4)\right)$

${ }^{13}$ C NMR: $\quad\left(400 \mathrm{MHz}, \mathrm{CDCl}_{3}\right)$

$138.11(\mathrm{C}(6)), 136.51(\mathrm{C}(9)), 128.06(\mathrm{C}(7)), 127.79(\mathrm{C}(8)), 113.75 \quad(\mathrm{C}(2)), 77.67$ (C(5)), 58.64 (C(3)), 58.54 (C(3)), 43.56 (C(1)), 20.99 (C(10)), 20.78 (C(4)), $16.95(\mathrm{C}(4))$

IR: $\quad\left(\mathrm{CHCl}_{3}\right)$

3614 (w), 3489 (br), 3010 (s), 2939 (s), 2836 (m), 1514 (w), 1471 (m), 1448 (w), 1390 (w), 1184 (m), 1104 (s), 1070 (s), 1015 (w), 953 (w), 830 (w), 762 (w)

MS: $\quad(\mathrm{FI})$

$238\left(\mathrm{M}^{+}, \quad 2\right), \quad 206 \quad\left(\mathrm{M}^{+}-\mathrm{MeOH}, \quad 14\right), \quad 120 \quad\left(\mathrm{CH}_{3} \mathrm{C}_{6} \mathrm{H}_{5} \mathrm{CHO}, \quad 40\right), \quad 86$ $\left(\left(\mathrm{CH}_{3}\right)_{2} \mathrm{CCHOMe,} \mathrm{100)}\right.$

Opt. Rot.: $\quad[\alpha]_{\mathrm{D}}^{24}-7.38(c=1.02, \mathrm{EtOH})$

TLC: $\quad R_{f} 0.29$ (hexane/EtOAc, 5/1) [silica gel, DNP]

SFC: $\quad(R)-4 b, t_{\mathrm{R}} 2.74 \min (72.8 \%) ;(S)-4 b, t_{\mathrm{R}} 3.30 \mathrm{~min}(27.2 \%)$ (Column: OD, MeOH $5 \%$, pressure $150 \mathrm{psi}$, flow $3.0 \mathrm{~mL} / \mathrm{min}$ )

Analysis: $\quad \mathrm{C}_{14} \mathrm{H}_{22} \mathrm{O}_{3}(238.32)$
Calcd:
C, 70.56;
$\mathrm{H}, 9.30 \%$
Found:
C, 70.29;
$\mathrm{H}, 9.54 \%$ 


\section{(R)-(2,2-Dimethoxy-1,1-dimethylethyl)-4-methoxyphenylmethanol (4c)}
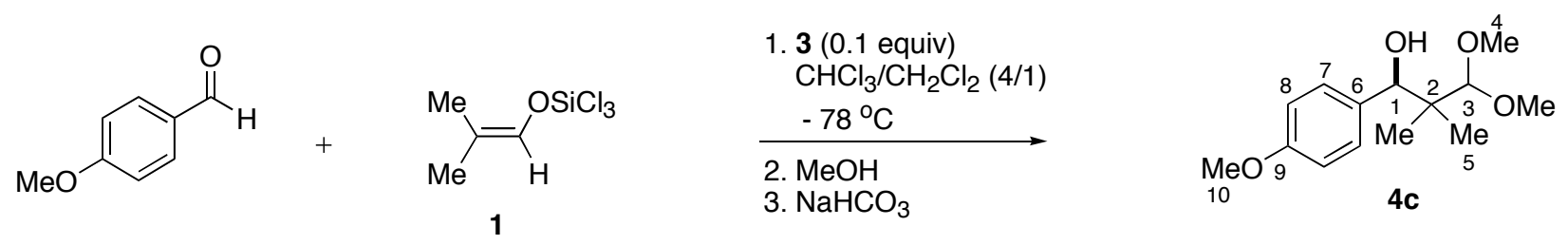

Following General Procedure I, a solution of 3 (84.3 mg, $0.1 \mathrm{mmol}, 0.1$ equiv), trichlorosilyl enolate 1 (226.1 mg, $1.1 \mathrm{mmol}, 1.1$ equiv), and anisaldehyde (122 $\mu \mathrm{L}, 1.0 \mathrm{mmol})$ in $4 \mathrm{~mL}$ of $\mathrm{CHCl}_{3} / \mathrm{CH}_{2} \mathrm{Cl}_{2}$ (4/1) was stirred at $-78{ }^{0} \mathrm{C}$ under nitrogen for $20 \mathrm{~h}$. Workup and purification by silica gel column chromatography using hexanes/ethyl acetate, 3/1 as eluent provided the desired aldol product, which was further purified by bulb-to-bulb distillation to afford $234.0 \mathrm{mg}(92 \%)$ of $\mathbf{4 c}$ as a clear, colorless oil.

Data for 4c

bp: $\quad 160{ }^{\circ} \mathrm{C}(0.02 \mathrm{mmHg}, \mathrm{ABT})$

1ㅁN NMR: $\quad\left(400 \mathrm{MHz}, \mathrm{CDCl}_{3}\right)$

$7.24(\mathrm{~d}, J=8.5,2 \mathrm{H}, 2 \times \mathrm{HC}(7)), 6.85(\mathrm{~d}, J=8.5,2 \mathrm{H}, 2 \times \mathrm{HC}(8)), 4.73$ (s, $1 \mathrm{H}$, $\mathrm{HC}(5)), 4.05$ (s, $1 \mathrm{H}, \mathrm{H}(\mathrm{C}(2)), 3.81$ (s, $\left.3 \mathrm{H}, \mathrm{H}_{3} \mathrm{C}(10)\right), 3.58$ (s, $\left.3 \mathrm{H}, \mathrm{H}_{3} \mathrm{C}(3)\right), 3.56$ (s, 3 H, H $\left.\mathrm{H}_{3}(3)\right), 0.92$ (s, $\left.3 \mathrm{H}, \mathrm{H}_{3} \mathrm{C}(4)\right), 0.75$ (s, $\left.3 \mathrm{H}, \mathrm{H}_{3} \mathrm{C}(4)\right)$

${ }^{13} \underline{\mathrm{C} \mathrm{NMR}}: \quad\left(400 \mathrm{MHz}, \mathrm{CDCl}_{3}\right)$

$158.66(\mathrm{C}(9)), 133.32(\mathrm{C}(6)), 128.97(\mathrm{C}(8)), 114.00(\mathrm{C}(7)), 112.81 \quad(\mathrm{C}(2)), 77.46$ $(\mathrm{C}(5)), 58.73(\mathrm{C}(3)), 58.68(\mathrm{C}(3)), 55.17$ (C(10)), 43.72 (C(1)), 21.06 (C(4)), $16.93(\mathrm{C}(5))$

IR: $\quad\left(\mathrm{CHCl}_{3}\right)$

3612 (w), 3487 (br), 3008 (s), 2968 (s), 2939 (s), 2837 (s), 1612 (m), 1514 (s), 1466 (m), 1444 (w), 1390 (w), 1304 (w), 1245 (s), 1174 (m), 1103 (s), 1070 (s), $1014(\mathrm{w}), 955(\mathrm{w}), 839(\mathrm{~m})$

MS: $\quad(\mathrm{FI})$

$254\left(\mathrm{M}^{+}, 100\right), 294(5), 136\left(\mathrm{CH}_{3} \mathrm{OC}_{6} \mathrm{H}_{5} \mathrm{CHO}, 5\right), 86\left(\left(\mathrm{CH}_{3}\right)_{2} \mathrm{CCHOMe}, 11\right)$

Opt. Rot.: $\quad[\alpha]_{\mathrm{D}}^{24}-7.59(c=0.90, \mathrm{EtOH})$

TLC: $\quad R_{f} 0.32$ (hexane/EtOAc, 5/1) [silica gel, DNP] 
SFC: $\quad(R)-4 c, t \mathrm{R} 2.53 \min (75.3 \%) ;(S)-4 \mathbf{c}, t_{\mathrm{R}} 3.21 \mathrm{~min}(24.7 \%)$ (Column: OD, MeOH $8 \%$, pressure $150 \mathrm{psi}$, flow $3.0 \mathrm{~mL} / \mathrm{min}$ )

Analysis: $\quad \mathrm{C}_{14} \mathrm{H}_{22} \mathrm{O} 4(254.32)$

$\begin{array}{lll}\text { Calcd: } & \text { C, 66.12; } & \text { H, 8.72\% } \\ \text { Found: } & \text { C }, 66.36 ; & \text { H }, 8.86 \%\end{array}$

(R)-(2,2-Dimethoxy-1,1-dimethylethyl)- 3,4,5-trimethoxyphenylmethanol (4d)<smiles>COc1cc(C=O)cc(OC)c1OC</smiles><smiles>CC(C)=COCCO</smiles>
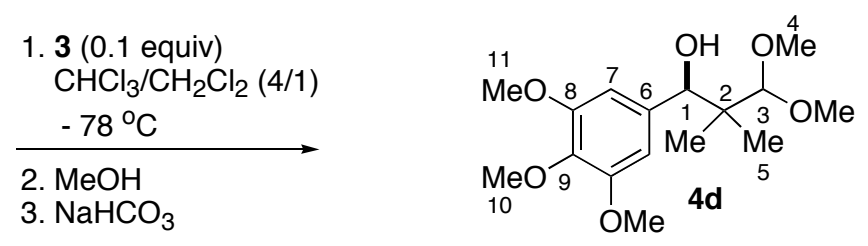

Following General Procedure I, a solution of 3 (84.3 mg, $0.1 \mathrm{mmol}, 0.1$ equiv), trichlorosilyl enolate 1 (226.1 mg, $1.1 \mathrm{mmol}, 1.1$ equiv), and 3,4,5- trimethoxybenzaldehyde (196.3 mg, $1.0 \mathrm{mmol}$ ) in $4 \mathrm{~mL}$ of $\mathrm{CHCl}_{3} / \mathrm{CH}_{2} \mathrm{Cl}_{2}$ (4/1) was stirred at $-78{ }^{\circ} \mathrm{C}$ under nitrogen for 28 h. Workup and purification by silica gel column chromatography using hexanes/ethyl acetate $(2 / 1)$ as eluent provided the desired aldol product, which was further purified by bulb to bulb distillation to afford $251.5 \mathrm{mg}(80 \%)$ of $\mathbf{4 d}$ as clear, colorless oil.

Data for 4d

bp: $\quad 160{ }^{\circ} \mathrm{C}(0.02 \mathrm{mmHg}, \mathrm{ABT})$

1슬 $: \quad\left(400 \mathrm{MHz}, \mathrm{CDCl}_{3}\right)$

6.55 (s, $2 \mathrm{H}, \mathrm{HC}(7)), 4.72(\mathrm{~d}, J=1.7,1 \mathrm{H}, \mathrm{HC}(5)), 4.05$ (s, $1 \mathrm{H}, \mathrm{HC}(2)), 3.89$ (d, $J$ $=1.7,1 \mathrm{H}, \mathrm{OH}), 3.85\left(\mathrm{~s}, 6 \mathrm{H}, 2 \times \mathrm{H}_{3} \mathrm{C}(11)\right), 3.83$ (s, $\left.3 \mathrm{H}, \mathrm{H}_{3} \mathrm{C}(10)\right), 3.58$ (s, $3 \mathrm{H}$, $\left.\mathrm{H}_{3} \mathrm{C}(3)\right), 3.57\left(\mathrm{~s}, 3 \mathrm{H}, \mathrm{H}_{3} \mathrm{C}(3)\right), 0.95\left(\mathrm{~s}, 3 \mathrm{H}, \mathrm{H}_{3} \mathrm{C}(4)\right), 0.76\left(\mathrm{~s}, 3 \mathrm{H}, \mathrm{H}_{3} \mathrm{C}(4)\right)$

${ }^{13} \underline{\mathrm{C} \mathrm{NMR}}: \quad\left(400 \mathrm{MHz}, \mathrm{CDCl}_{3}\right)$

$152.34(\mathrm{C}(8)), 136.86(\mathrm{C}(9)), 136.77(\mathrm{C}(6)), 114.22(\mathrm{C}(7)), 105.00 \quad(\mathrm{C}(2))$, 77.79(C(5)), 60.79 (C(11)), 58.79 (C(3)), 58.76 (C(3)), 55.99 (C(10)), 43.63 $(\mathrm{C}(1)), 21.41(\mathrm{C}(4)), 17.07(\mathrm{C}(4))$ 
IR: $\quad\left(\mathrm{CHCl}_{3}\right)$

3610 (w), 3481 (br), 3010 (s), 2966 (s), 2941 (s), 2837 (m), 1593 (s), 1506 (m), 1464 (s), 1421 (m), 1327 (m), 1236 (m), 1186 (w), 1130 (s), 1105 (m), 1070 (m), $1003(\mathrm{w}), 955(\mathrm{w}), 852(\mathrm{w})$

MS: $\quad(\mathrm{FI})$

$314\left(\mathrm{M}^{+}, 100\right), 282\left(\mathrm{M}^{+}-\mathrm{MeOH}, 2\right), 220(2)$

Opt. Rot.: $\quad[\alpha]_{\mathrm{D}}^{24}-1.48(c=0.85, \mathrm{EtOH})$

TLC: $\quad R_{f} 0.32$ (hexane/EtOAc, 2/1) [silica gel, DNP]

SFC: $\quad(R)-4 d, t_{\mathrm{R}} 4.97 \min (87.5 \%) ;(S)-4 d, t_{\mathrm{R}} 6.09 \min (12.5 \%)$ (Column: OD, MeOH $5 \%$, pressure $150 \mathrm{psi}$, flow $3.0 \mathrm{~mL} / \mathrm{min}$ )

Analysis: $\quad \mathrm{C}_{16} \mathrm{H}_{26} \mathrm{O}_{6}(314.37)$

Calcd: $\quad$ C, $61.13 ; \quad \mathrm{H}, 8.34 \%$

Found: $\quad \mathrm{C}, 61.16 ; \quad \mathrm{H}, 8.44 \%$

\section{(R)-(2,2-Dimethoxy-1,1-dimethylethyl)-4-chlorophenylmethanol (4e)}
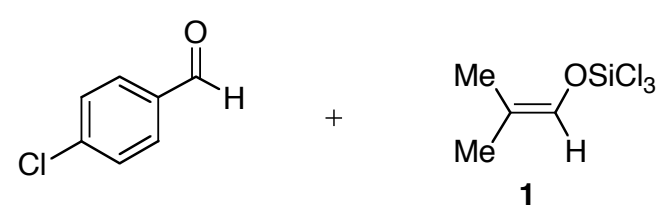
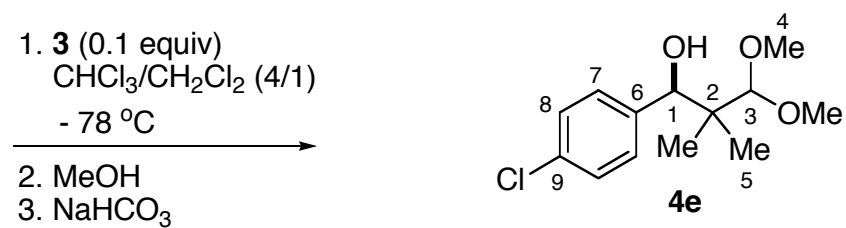

Following General Procedure I, a solution of 3 (84.3 mg, $0.1 \mathrm{mmol}, 0.1$ equiv), trichlorosilyl enolate 1 (226.1 mg, $1.1 \mathrm{mmol}, 1.1$ equiv), and 4-chlorobenzaldehyde (140.6 mg, $1.0 \mathrm{mmol})$ in $4 \mathrm{~mL}$ of $\mathrm{CHCl}_{3} / \mathrm{CH}_{2} \mathrm{Cl}_{2}(4 / 1)$ was stirred at $-78{ }^{\circ} \mathrm{C}$ under nitrogen for $8 \mathrm{~h}$. Workup and purification by silica gel column chromatography using hexanes/ethyl acetate (5/1) as eluent provided the desired aldol product, which was further purified by bulb-to-bulb distillation to afford $219 \mathrm{mg}(85 \%)$ of $\mathbf{4 e}$ as clear, colorless oil.

\section{Data for 4e}

bp: $\quad 160{ }^{\circ} \mathrm{C}(0.02 \mathrm{mmHg}, \mathrm{ABT})$ 
$1_{\text {H NMR: }} \quad\left(400 \mathrm{MHz}, \mathrm{CDCl}_{3}\right)$

7.25-7.03 (m, 4 H, Aryl-H), 4.79 (d, $J=2.2,1$ H, HC(5)), 4.04 (s, $1 \mathrm{H}, \mathrm{H}(\mathrm{C}(2))$, 3.99 (d, $J=2.2,1 \mathrm{H}, \mathrm{OH}$ ), 3.59 (s, $\left.3 \mathrm{H}, \mathrm{H}_{3} \mathrm{C}(3)\right), 3.57$ (s, $\left.3 \mathrm{H}, \mathrm{H}_{3} \mathrm{C}(3)\right), 0.90$ (s, 3 $\left.\mathrm{H}, \mathrm{H}_{3} \mathrm{C}(4)\right), 0.73$ (s, $\left.3 \mathrm{H}, \mathrm{H}_{3} \mathrm{C}(4)\right)$

${ }^{13}$ C NMR: $\quad\left(400 \mathrm{MHz}, \mathrm{CDCl}_{3}\right)$

$138.61(\mathrm{C}(6)), 132.79(\mathrm{C}(9)), 129.35(\mathrm{C}(7)), 127.60(\mathrm{C}(8)), 114.20 \quad(\mathrm{C}(2)), 77.10$ $(\mathrm{C}(5)), 58.79(\mathrm{C}(3)), 43.52(\mathrm{C}(1)), 21.42(\mathrm{C}(4)), 16.78(\mathrm{C}(4))$

IR: $\quad\left(\mathrm{CHCl}_{3}\right)$

3610 (w), 3479 (br), 3010 (s), 2970 (s), 2941 (s), 2837 (m), 1597 (w), 1491 (m), 1471 (m), 1448 (w), 1390 (w), 1190 (w), 1103 (s), 1070 (s), 1014 (m), 839 (m)

MS: $\quad(\mathrm{FI})$

$259\left(\mathrm{M}^{+}+\mathrm{H}, 21\right), 226\left(\mathrm{M}^{+}-\mathrm{MeOH}, 19\right), 183(65), 140\left(\mathrm{ClC}_{6} \mathrm{H}_{5} \mathrm{CHO}, 26\right), 86$ $\left(\left(\mathrm{CH}_{3}\right)_{2} \mathrm{CCHOMe}, 100\right)$

Opt. Rot.: $\quad[\alpha]_{D}^{24}-11.31(c=1.00, \mathrm{EtOH})$

TLC: $\quad R_{f} 0.26$ (hexane/EtOAc, 5/1) [silica gel, DNP]

SFC: $\quad(S)-4 \mathrm{e}, t_{\mathrm{R}} 3.70 \mathrm{~min}(11.0 \%) ;(R)-4 \mathrm{e}, t_{\mathrm{R}} 5.01 \mathrm{~min}(89.0 \%)$ (Column: AS, $\mathrm{MeOH}$ $1 \%$, pressure $150 \mathrm{psi}$, flow $3.0 \mathrm{~mL} / \mathrm{min}$ )

Analysis: $\quad \mathrm{C}_{13} \mathrm{H}_{19} \mathrm{ClO}_{3}(258.74)$

Calcd: $\quad$ C, $60.35 ; \quad \mathrm{H}, 7.40 ; \quad \mathrm{Cl}, 13.70 \%$

Found: $\quad \mathrm{C}, 60.27 ; \quad \mathrm{H}, 7.73 ; \quad \mathrm{Cl}, 13.45 \%$

\section{(R)-(2,2-Dimethoxy-1,1-dimethylethyl)-4-trifluoromethylphenylmethanol (4f)}<smiles>O=Cc1ccc(C(F)(F)F)cc1</smiles>
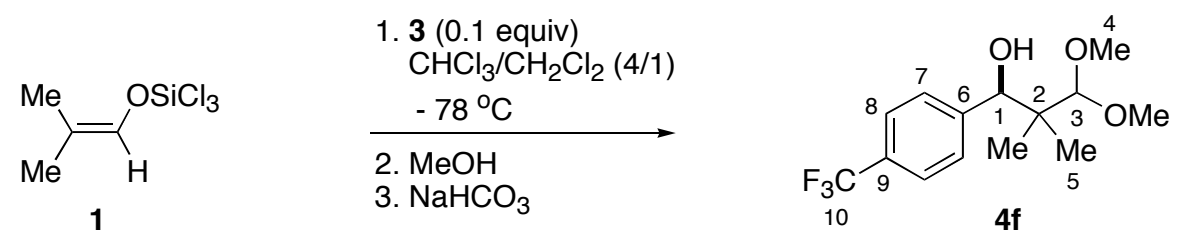

Following General Procedure I, a solution of 3 ( $84.3 \mathrm{mg}, 0.1 \mathrm{mmol}, 0.1$ equiv), trichlorosilyl enolate 1 (226.1 mg, $1.1 \mathrm{mmol}, 1.1$ equiv), and 4-trifluoromethylbenzaldehyde $(137 \mu \mathrm{L}, 1.0 \mathrm{mmol})$ in $4 \mathrm{~mL}$ of $\mathrm{CHCl}_{3} / \mathrm{CH}_{2} \mathrm{Cl}_{2}(4 / 1)$ was stirred at $-78{ }^{\circ} \mathrm{C}$ under nitrogen for $8 \mathrm{~h}$. Workup and purification by silica gel column chromatography using hexanes/ethyl acetate (5/1) 
as eluent provided the desired aldol product, which was further purified by recrystallization from hexanes/ethyl acetate (6/1) to afford $128 \mathrm{mg}(86 \%)$ of $\mathbf{4 f}$ as white crystals.

Data for $\mathbf{4 f}$

mp: $\quad 77^{\circ} \mathrm{C}$

1ㅁN $\underline{\text { NMR: }} \quad\left(400 \mathrm{MHz}, \mathrm{CDCl}_{3}\right)$

$7.56(\mathrm{~d}, J=8.1,2 \mathrm{H}, \mathrm{HC}(8)), 7.44(\mathrm{~d}, J=8.3,2 \mathrm{H}, \mathrm{HC}(7)), 4.87(\mathrm{~d}, J=2.0,1 \mathrm{H}$, $\mathrm{HC}(5)), 4.09$ (d, J = 2.0, $1 \mathrm{H}, \mathrm{OH}$ ), 4.05 (s, $1 \mathrm{H}, \mathrm{H}(\mathrm{C}(2)), 3.60$ (s, $\left.3 \mathrm{H}, \mathrm{H}_{3} \mathrm{C}(3)\right)$, $3.58\left(\mathrm{~s}, 3 \mathrm{H}, \mathrm{H}_{3} \mathrm{C}(3)\right), 0.91\left(\mathrm{~s}, 3 \mathrm{H}, \mathrm{H}_{3} \mathrm{C}(4)\right), 0.74$ (s, $\left.3 \mathrm{H}, \mathrm{H}_{3} \mathrm{C}(4)\right)$

${ }^{13} \underline{\mathrm{C} \mathrm{NMR}}: \quad\left(400 \mathrm{MHz}, \mathrm{CDCl}_{3}\right)$

$145.18(\mathrm{C}(9)), 129.30(\mathrm{C}(10)), 128.35(\mathrm{C}(6)), 124.39(\mathrm{C}(8)), 124.34(\mathrm{C}(7))$, 114.30, (C(2)), $77.16(\mathrm{C}(5)), 58.84$ (C(3)), 43.50 (C(1)), 21.57 (C(4)), 16.76 $(\mathrm{C}(4))$

IR: $\quad\left(\mathrm{CHCl}_{3}\right)$

3620 (w), 3479 (br), 3010 (s), 2970 (s), 2939 (s), 1620 (w), 1471 (w), 1413 (w), 1365 (w), 1325 (s), 1211 (w), 1167 (s), 1128 (s), 1113 (s), 1068 (s), 1018 (w), 852 (w)

$\underline{\mathrm{MS}}: \quad(\mathrm{FI})$

$293\left(\mathrm{M}^{+}+\mathrm{H}, \quad 0.2\right), \quad 217 \quad(6), \quad 183 \quad(65), \quad 174 \quad\left(\mathrm{CF}_{3} \mathrm{C}_{6} \mathrm{H}_{5} \mathrm{CHO}, \quad 26\right), \quad 86$ $\left(\left(\mathrm{CH}_{3}\right)_{2} \mathrm{CCHOMe}, 100\right)$

Opt. Rot.: $\quad[\alpha]_{\mathrm{D}}^{24}-6.15(c=1.58, \mathrm{EtOH})$

TLC: $\quad R f 0.24$ (hexane/EtOAc, 5/1) [silica gel, DNP]

SFC: $\quad(S)-4 \mathbf{f}, t \mathrm{R} 1.85 \min (10.1 \%) ;(R)-4 \mathbf{f}, t \mathrm{R} 2.24 \min (89.9 \%)$ (Column: AS, MeOH $1 \%$, pressure $150 \mathrm{psi}$, flow $3.0 \mathrm{~mL} / \mathrm{min}$ )

Analysis: $\quad \mathrm{C}_{14} \mathrm{H}_{19} \mathrm{~F}_{3} \mathrm{O}_{3}(292.29)$

$\begin{array}{llll}\text { Calcd: } & \text { C }, 57.53 ; & \text { H, 6.55; } & \text { F, 19.50\% } \\ \text { Found: } & \text { C , 57.31; } & \text { H, 6.80; } & \text { F, 19.45\% }\end{array}$




\section{(R)-(2,2-Dimethoxy-1,1-dimethylethyl)-4-nitrophenylmethanol (4g)}<smiles>O=Cc1ccc([N+](=O)[O-])cc1</smiles>
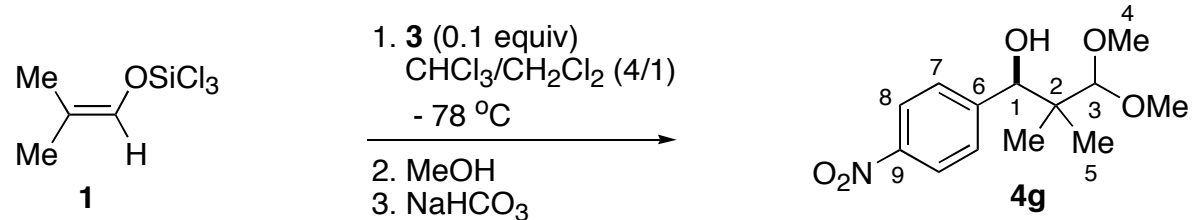

Following General Procedure I, a solution of 3 (84.3 mg, $0.1 \mathrm{mmol}, 0.1$ equiv), trichlorosilyl enolate 1 (226.1 mg, $1.1 \mathrm{mmol}, 1.1$ equiv), and 4-nitrobenzaldehyde (151.1 mg, 1.0 mmol) in $4 \mathrm{~mL}$ of $\mathrm{CHCl}_{3} / \mathrm{CH}_{2} \mathrm{Cl}_{2}(4 / 1)$ was stirred at $-78{ }^{\circ} \mathrm{C}$ under nitrogen for $8 \mathrm{~h}$. Workup and purification by silica gel column chromatography using hexanes/ethyl acetate, 3/1 as eluent provided the desired aldol product, which was further purified by recrystallization from hexanes/ethyl acetate (4/1) to afford $240 \mathbf{m g}(89 \%)$ of $\mathbf{4 g}$ as white crystals.

Data for $\mathbf{4 g}$

mp: $\quad 87^{\circ} \mathrm{C}$

1ㅁN NMR: $\quad\left(400 \mathrm{MHz}, \mathrm{CDCl}_{3}\right)$

$8.17(\mathrm{~d}, J=8.9,2 \mathrm{H}, \mathrm{HC}(8)), 7.51(\mathrm{~d}, J=8.5,2 \mathrm{H}, \mathrm{HC}(7)), 4.95$ (d, $J=1.7,1 \mathrm{H}$, $\mathrm{HC}(5)), 4.23$ (d, J = 1.7, $1 \mathrm{H}, \mathrm{OH}$ ), 4.05 (s, $1 \mathrm{H}, \mathrm{H}(\mathrm{C}(2)), 3.59$ (s, $\left.3 \mathrm{H}, \mathrm{H}_{3} \mathrm{C}(3)\right)$, 3.58 (s, $\left.3 \mathrm{H}, \mathrm{H}_{3} \mathrm{C}(3)\right), 0.90$ (s, $\left.3 \mathrm{H}, \mathrm{H}_{3} \mathrm{C}(4)\right), 0.73$ (s, $\left.3 \mathrm{H}, \mathrm{H}_{3} \mathrm{C}(4)\right)$

${ }^{13}$ C NMR: $\quad\left(400 \mathrm{MHz}, \mathrm{CDCl}_{3}\right)$

$148.76(\mathrm{C}(9)), 147.11(\mathrm{C}(6)), 128.86(\mathrm{C}(8)), 122.63$ (C(7)), $114.30(\mathrm{C}(3)), 76.75$

(C(5)), 58.93 (C(3)), 58.85 (C(3)), 43.49 (C(1)), 21.82 (C(4)), 16.69 (C(4))

IR: $\quad\left(\mathrm{CHCl}_{3}\right)$

3469 (br), 3014 (m), 2970 (m), 2914 (m), 2837 (m), 1606 (w), 1519 (s), 1471 (w), 1348 (s), 1190 (w), 1105 (m), 1070 (s), 990 (w), 862 (w)

MS: (FI)

$270\left(\mathrm{M}^{+}+\mathrm{H}, 11\right), 238\left(\mathrm{M}^{+}-\mathrm{MeOH}, 3\right), 117$ (12), $151\left(\mathrm{NO}_{2} \mathrm{C}_{6} \mathrm{H}_{5} \mathrm{CHO}, 2\right), 86$ $\left(\left(\mathrm{CH}_{3}\right)_{2} \mathrm{CCHOMe}, 100\right)$

Opt. Rot.: $\quad[\alpha]_{\mathrm{D}}^{24}+25.70\left(c=0.71, \mathrm{CHCl}_{3}\right)$

TLC: $\quad R_{f} 0.29$ (hexane/EtOAc, 2/1) [silica gel]

SFC: $\quad(S)-4 g, t \mathrm{R} 8.53 \min (9.0 \%) ;(R)-4 g, t \mathrm{R} 9.04 \min (91.0 \%)($ Column: $\mathrm{AD}, \mathrm{MeOH}$ $2.5 \%$, pressure $150 \mathrm{psi}$, flow $3.0 \mathrm{~mL} / \mathrm{min}$ ) 
Analysis: $\quad \mathrm{C}_{13} \mathrm{H}_{19} \mathrm{NO}_{5}(269.29)$

$\begin{array}{llll}\text { Calcd: } & \text { C , 57.98; } & \text { H, 7.11; } & \text { N, 5.20\% } \\ \text { Found: } & \text { C , 57.86; } & \text { H, 7.11; } & \text { N, 5.26\% }\end{array}$

\section{2. ${ }^{12} \mathrm{C} /{ }^{13} \mathrm{C}$ Kinetic Isotope at Natural Abundance ${ }^{3}$}

\section{General Procedure II}

\section{(R)-(2,2-Dimethoxy-1,1-dimethylethyl)- 3,4,5-trimethoxyphenylmethanol (4d)}
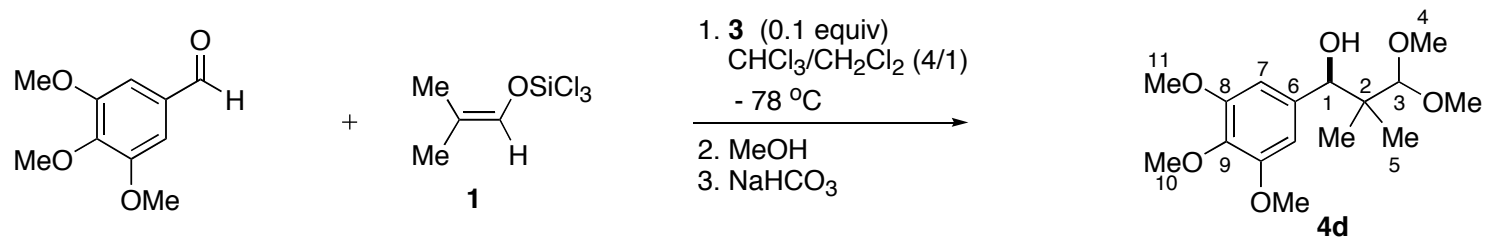

To a stirred solution of $\mathbf{3}$ (505.8 $\mathrm{mg}, 0.6 \mathrm{mmol}, 0.1$ equiv) and trimethoxybenzaldehyde (58.9 mg, $0.3 \mathrm{mmol}, 0.05$ equiv) in $4 \mathrm{~mL}$ of chloroform/methylene chloride (4/1) at $-78{ }^{0} \mathrm{C}$ was added dropwise the enolate 1 ( $1.2 \mathrm{~g}, 6.0 \mathrm{mmol}, 1$ equiv). The reaction mixture was stirred at - 78 ${ }^{0} \mathrm{C}$ under $\mathrm{N}_{2}$ for $32 \mathrm{~h}$. Dry methanol $(18 \mathrm{~mL})$ was then added dropwise at $-78{ }^{0} \mathrm{C}$, and the resultant mixture was stirred for $45 \mathrm{~min}$. The solution was allowed to warm to room temperature and was then quickly poured into a cold aqueous sodium bicarbonate solution (40 mL). The methanolic solution was stirred at room temperature for $1 \mathrm{~h}$ and then filtered through celite. The aqueous layer was separated and extracted with methylene chloride ( 3 x $25 \mathrm{~mL})$. The combined organic layers were dried over sodium sulfate, filtered, and concentrated in vacuo. Silica gel column chromatography (hexanes/ethyl acetate, 2/1) afforded $84.9 \mathrm{mg}$ (90\%) of 4d as a clear, colorless oil.

\section{Data for 4d}

${ }^{1}$ H NMR: $\quad\left(400 \mathrm{MHz}, \mathrm{CDCl}_{3}\right)$

6.55 (s, $2 \mathrm{H}, \mathrm{HC}(7)), 4.72(\mathrm{~d}, J=1.7,1 \mathrm{H}, \mathrm{HC}(1)), 4.05$ (s, $1 \mathrm{H}, \mathrm{HC}(3)), 3.89$ (d, $J$ $=1.7,1 \mathrm{H}, \mathrm{OH}), 3.85\left(\mathrm{~s}, 6 \mathrm{H}, 2 \mathrm{x} \mathrm{H}_{3} \mathrm{C}(11)\right), 3.83\left(\mathrm{~s}, 3 \mathrm{H}, \mathrm{H}_{3} \mathrm{C}(10)\right), 3.58(\mathrm{~s}, 3 \mathrm{H}$, $\left.\mathrm{H}_{3} \mathrm{C}(4)\right), 3.57$ (s, $\left.3 \mathrm{H}, \mathrm{H}_{3} \mathrm{C}(4)\right), 0.95$ (s, $\left.3 \mathrm{H}, \mathrm{H}_{3} \mathrm{C}(5)\right), 0.76$ (s, $\left.3 \mathrm{H}, \mathrm{H}_{3} \mathrm{C}(5)\right)$

${ }^{13}$ C NMR: $\quad(400 \mathrm{MHz}, \mathrm{CDCl} 3)$

$152.34(\mathrm{C}(8)), 136.86(\mathrm{C}(9)), 136.77(\mathrm{C}(6)), 114.22(\mathrm{C}(7)), 105.00 \quad(\mathrm{C}(3))$,

77.79(C(1)), $60.79(\mathrm{C}(11)), 58.79(\mathrm{C}(4)), 58.76(\mathrm{C}(4)), 55.99$ (C(10)), 43.63

$(\mathrm{C}(2)), 21.41(\mathrm{C}(5)), 17.07(\mathrm{C}(5))$

TLC: $\quad R_{f} 0.32$ (hexane/EtOAc, 2/1) [silica gel, DNP] 
All of the steps in the NMR analysis were were carried out as described in the text on a Varian Unity $500 \mathrm{MHz}$ spectrometer. The following values were determined experimentally following standard NMR procedures.

2d: 90 degree pulse width $=8 \mathrm{~s}$; relaxation time: $11.6 \mathrm{~s}$; delay time: $93 \mathrm{~s}$; acquistion time about $30 \mathrm{~h}$. Integration for each signal was determined by the half-height of the line-width of the intergrated peak multiplied by ten on each side.

2f: 90 degree pulse width $=9$ s; relaxation time:10.1; delay time: $81 \mathrm{~s}$; acquisition time about $30 \mathrm{~h}$. Integration was determined as described above.

The ${ }^{13} \mathrm{C}$ spectra of the sample and the standard were integrated five times and the average integration values are summarized in Table 1 and Table 2 below. The calculation of ${ }^{12} \mathrm{C} /{ }^{13} \mathrm{C}$ kinetic isotope effects was done according to the previously reported procedure, ${ }^{3}$ and the results are presented in Table 3.

Table 1. Average integration for the sample<smiles>COc1cc(C(O)C(C)(C)C(OC)OC)cc(OC)c1OC</smiles>

\begin{tabular}{|c|c|c|c|c|}
\hline Spectrum \# & $\begin{array}{c}\mathrm{C}_{\mathrm{a}} \\
152.28(\delta)\end{array}$ & $\begin{array}{c}\mathrm{C}_{\mathrm{b}} \\
105.01\end{array}$ & $\begin{array}{c}\mathrm{C}_{\mathrm{c}} \\
55.89\end{array}$ & $\begin{array}{c}\mathrm{C}_{\mathrm{d}} \\
43.57\end{array}$ \\
\hline 1 & 0.5662 & 1.017 & 1.000 & 0.4868 \\
\hline 2 & 0.5661 & 1.019 & 1.000 & 0.4867 \\
\hline 3 & 0.5659 & 1.018 & 1.000 & 0.4865 \\
\hline 4 & 0.5659 & 1.018 & 1.000 & 0.4865 \\
\hline 5 & 0.5662 & 1.017 & 1.000 & 0.4860 \\
\hline Ave. integration & 0.5661 & 1.018 & 1.000 & 0.4865 \\
\hline standard deviation & 0.0001 & 0.0000 & 0.0000 & 0.0003 \\
\hline
\end{tabular}

Preparation of the standard 4d, Following General Procedure II except using enolate 1 and trimethoxybenzaldehyde in about 1:1 ratio. A solution of 3 ( $84.3 \mathrm{mg}, 0.1 \mathrm{mmol}, 0.1 \mathrm{equiv})$, trichlorosilyl enolate 1 (226.1 mg, $1.1 \mathrm{mmol}, 1.1$ equiv), and 3,4,5- trimethoxybenzaldehyde (196.3 mg, $1.0 \mathrm{mmol}$ ) in $4 \mathrm{~mL}$ of $\mathrm{CHCl}_{3} / \mathrm{CH}_{2} \mathrm{Cl}_{2}$ (4/1) was stirred at $-78{ }^{\circ} \mathrm{C}$ under nitrogen for 28 h. Workup and purification by silica gel column chromatography using hexanes/ethyl acetate $(2 / 1)$ as eluent provided the desired aldol product, which was further purified by bulb to bulb distillation to afford $251.5 \mathrm{mg}(80 \%)$ of $\mathbf{4 d}$ as clear, colorless oil. 
Table 2. Average integration for the standard

\begin{tabular}{|c|c|c|c|c|}
\hline spectrum \# & $\begin{array}{c}\mathrm{C}_{\mathrm{a}} \\
152.28(\delta)\end{array}$ & $\begin{array}{c}\mathrm{C}_{\mathrm{b}} \\
105.01\end{array}$ & $\begin{array}{c}\mathrm{C}_{\mathrm{c}} \\
55.89\end{array}$ & $\begin{array}{c}\mathrm{C}_{\mathrm{d}} \\
43.57\end{array}$ \\
\hline 1 & 0.5711 & 1.017 & 1.000 & 0.4925 \\
\hline 2 & 0.5708 & 1.017 & 1.000 & 0.4923 \\
\hline 3 & 0.5658 & 1.018 & 1.000 & 0.4928 \\
\hline 4 & 0.5681 & 1.017 & 1.000 & 0.4934 \\
\hline 5 & 0.5684 & 1.018 & 1.000 & 0.4925 \\
\hline Ave. integration & 0.5688 & 1.017 & 1.000 & 0.4927 \\
\hline standard deviation & 0.0022 & 0.0004 & 0.0000 & 0.0004 \\
\hline
\end{tabular}

Table 3. ${ }^{12} \mathrm{C} /{ }^{13} \mathrm{C}$ kinetic isotope effects for $\mathbf{4} \mathbf{d}^{1}$<smiles>COc1cc(C(O)C(C)(C)C(C)(C)OC)cc(OC)c1OC</smiles>

\begin{tabular}{|lcccc|}
\hline & $\mathrm{C}_{\mathrm{a}}$ & $\mathrm{C}_{\mathrm{b}}$ & $\mathrm{C}_{\mathrm{c}}$ & $\mathrm{C}_{\mathrm{d}}$ \\
$\delta(\mathrm{ppm})$ & 152.28 & 105.01 & 55.89 & 43.57 \\
Standard & 0.568 & 1.017 & 1.000 & 0.493 \\
Sample & 0.566 & 1.018 & 1.000 & 0.486 \\
$\mathrm{KIE}=\mathrm{R}_{0} / \mathrm{R}$ & 1.004 & 0.999 & 1.000 & 1.014 \\
\hline
\end{tabular}

(R)-(2,2-Dimethoxy-1,1-dimethylethyl)-4-trifluoromethylphenylmethanol (4f)<smiles>O=C(O)c1ccc(C(F)(F)F)cc1</smiles><smiles>CC(C)=COCCO</smiles>

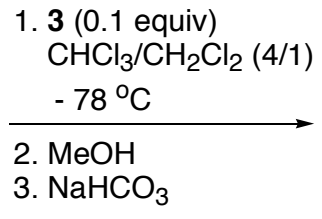<smiles>[Y6]C(O)(c1ccc(C(F)(F)F)cc1)C(C)(O)C(OC)OC</smiles>

Following General Procedure II, a solution of $\mathbf{3}$ (590 mg, $0.7 \mathrm{mmol}, 0.1$ equiv), trichlorosilyl enolate 1 ( $1.4 \mathrm{~g}, 7 \mathrm{mmol}, 1$ equiv), and 4-trifluoromethylbenzaldehyde (48 $\mu \mathrm{L}, 0.35$ mmol, 0.05 equiv) in $4 \mathrm{~mL}$ of $\mathrm{CHCl}_{3} / \mathrm{CH}_{2} \mathrm{Cl}_{2}$ was stirred at $-78{ }^{0} \mathrm{C}$ under nitrogen for $15 \mathrm{~h}$. 
Workup and purification by silica gel column chromatography using hexanes/ethyl acetate, 5/1 as eluent provided $92 \mathrm{mg}(90 \%)$ of $\mathbf{4 f}$ as a white solid.

\section{Data for $4 \mathbf{f}$}

1 $\underline{\text { H NMR: }} \quad\left(400 \mathrm{MHz}, \mathrm{CDCl}_{3}\right)$

$7.56(\mathrm{~d}, J=8.1,2 \mathrm{H}, \mathrm{HC}(8)), 7.44$ (d, $J=8.3,2 \mathrm{H}, \mathrm{HC}(7)), 4.87$ (d, $J=2.0,1 \mathrm{H}$, $\mathrm{HC}(1)), 4.09$ (d, J = 2.0, $1 \mathrm{H}, \mathrm{OH}$ ), 4.05 (s, $1 \mathrm{H}, \mathrm{H}(\mathrm{C}(3)), 3.60$ (s, $\left.3 \mathrm{H}, \mathrm{H}_{3} \mathrm{C}(4)\right)$, 3.58 (s, $\left.3 \mathrm{H}, \mathrm{H}_{3} \mathrm{C}(4)\right), 0.91$ (s, $\left.3 \mathrm{H}, \mathrm{H}_{3} \mathrm{C}(5)\right), 0.74$ (s, $\left.3 \mathrm{H}, \mathrm{H}_{3} \mathrm{C}(5)\right)$

${ }^{13}$ C NMR: $\quad\left(400 \mathrm{MHz}, \mathrm{CDCl}_{3}\right)$

145.18 (C(9)), 129.30 (C(10)), $128.35(\mathrm{C}(6)), 124.39$ (C(8)), $124.34(\mathrm{C}(7))$,

114.21 (C(3)), $77.16(\mathrm{C}(1)), 58.84(\mathrm{C}(4)), 43.50(\mathrm{C}(2)), 21.57(\mathrm{C}(5)), 16.76$ $(\mathrm{C}(5))$

TLC: $\quad R f 0.24$ (hexane/EtOAc, 5/1) [silica gel, DNP]

The analysis of the spectral data for compound $\mathbf{4 f}$ was performed in the same manner as previously described for compound 4d. The average integration values and ${ }^{12} \mathrm{C} /{ }^{13} \mathrm{C}$ kinetic isotope effects are provided in Tables 4, 5 and 6 respectively.

Table 4. Average integration for the sample<smiles>COC(OC)C(C)(C)C(O)c1ccc(C(F)(F)F)cc1</smiles>

\begin{tabular}{|c|c|c|c|c|}
\hline Spectrum \# & $\begin{array}{c}\mathrm{C}_{\mathrm{a}} \\
145.29(\delta)\end{array}$ & $\begin{array}{c}\mathrm{C}_{\mathrm{b}} \\
114.21\end{array}$ & $\begin{array}{c}\mathrm{C}_{\mathrm{c}} \\
43.48\end{array}$ & $\begin{array}{c}\mathrm{C}_{\mathrm{d}} \\
21.19\end{array}$ \\
\hline 1 & 0.531 & 1.115 & 0.988 & 1.000 \\
\hline 2 & 0.530 & 1.115 & 0.988 & 1.000 \\
\hline 3 & 0.530 & 1.115 & 0.988 & 1.000 \\
\hline 4 & 0.531 & 1.115 & 0.988 & 1.000 \\
\hline 5 & 0.531 & 1.114 & 0.988 & 1.000 \\
\hline Ave. integration & 0.531 & 1.115 & 0.988 & 1.000 \\
\hline standard deviation & 0.0005 & 0.0007 & 0.0000 & 0.0000 \\
\hline
\end{tabular}


Table 5. Average integration for the standard

\begin{tabular}{|c|c|c|c|c|}
\hline Spectrum \# & $\begin{array}{c}\mathrm{C}_{\mathrm{a}} \\
145.29(\delta)\end{array}$ & $\begin{array}{c}\mathrm{C}_{\mathrm{b}} \\
114.21\end{array}$ & $\begin{array}{c}\mathrm{C}_{\mathrm{c}} \\
43.48\end{array}$ & $\begin{array}{c}\mathrm{C}_{\mathrm{d}} \\
21.19\end{array}$ \\
\hline 1 & 0.531 & 1.119 & 1.004 & 1.000 \\
\hline 2 & 0.533 & 1.112 & 1.007 & 1.000 \\
\hline 3 & 0.533 & 1.110 & 1.006 & 1.000 \\
\hline 4 & 0.532 & 1.111 & 1.006 & 1.000 \\
\hline 5 & 0.533 & 1.111 & 1.007 & 1.000 \\
\hline Ave. integration & 0.5324 & 1.115 & 1.006 & 1.000 \\
\hline standard deviation & 0.0009 & 0.0036 & 0.0012 & 0.000 \\
\hline
\end{tabular}

Table 6: ${ }^{12} \mathrm{C} /{ }^{13} \mathrm{C}$ kinetic isotope effects for $\mathbf{4 f}$

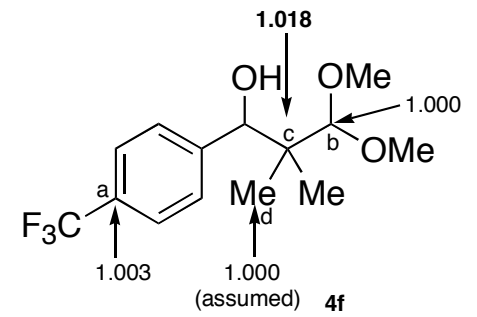

\begin{tabular}{|lcccc|}
\hline & $\mathrm{C}_{\mathrm{a}}$ & $\mathrm{C}_{\mathrm{b}}$ & $\mathrm{C}_{\mathrm{c}}$ & $\mathrm{C}_{\mathrm{d}}$ \\
$\delta(\mathrm{ppm})$ & 145.29 & 114.21 & 43.48 & 21.39 \\
Standard & 0.532 & 1.015 & 1.006 & 1.000 \\
Sample & 0.531 & 1.015 & 0.988 & 1.000 \\
$\mathrm{KIE}=\mathrm{R}_{0} / \mathrm{R}$ & 1.003 & 1.000 & 1.018 & 1.000 \\
\hline
\end{tabular}

\section{Arrhenius Studies}

The following experiments are kinetic runs for the aldol addition of enolate $\mathbf{1}$ to aldehyde 2d and $2 \mathrm{f}$ at various temperatures ranging from $-45{ }^{0} \mathrm{C}$ to $-73{ }^{0} \mathrm{C}$. Two kinetic runs were performed at each indicated temperature. The raw kinetic data and the graph of time versus 1/[aldehyde] are provided for each run. 


\section{Kinetic Data for 2d}

\section{General Procedure III}

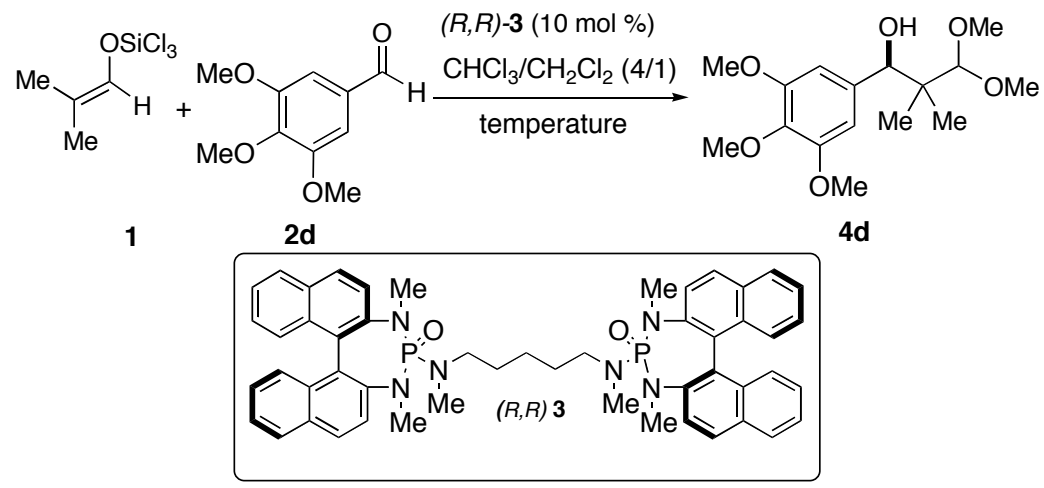

A dry reaction vessel was assembled to the reactIR probe and then purged with argon for $30 \mathrm{~min}$. Dry chloroform and dichloromethane $(4 / 1,2.0 \mathrm{~mL})$ were added and a background file was collected. In a separate flame dried flask, aldehyde $2 \mathbf{2 d}(41.2 \mathrm{mg}, 0.21 \mathrm{mmol})$ and the dimeric catalyst $(R, R)-3(17.7 \mathrm{mg}, 0.021 \mathrm{mmol})$ were weighed out and dissolved in the same solvent $(0.5 \mathrm{~mL})$. The resulting solution was transferred to the vessel using a syringe. The flask was rinsed with the solvent $(0.5 \mathrm{~mL})$ and the washings were transferred to the vessel in the same manner as before. The solution of the catalyst and aldehyde 2d was thoroughly mixed and cooled to the indicated temperature by internal monitoring. To this solution, enolate $\mathbf{1}(31.3 \mu \mathrm{L}$, $0.21 \mathrm{mmol}$ ) was added quickly. The disappearance of the aldehyde was monitored every one minute interval for $40 \mathrm{~min}$ at the indicated internal temperature and then incrementally thereafter until complete conversion.

\section{Temperature: $-45^{\circ} \mathrm{C}$, run 1}

Following General Procedure III, enolate $1(31.3 \mu \mathrm{L}, 0.21 \mathrm{mmol})$ was added to the solution of aldehyde $2 \mathbf{2 d}(41.2 \mathrm{mg}, 0.21 \mathrm{~mol})$ and the dimeric catalyst $(R, R)-3(17.7 \mathrm{mg}, 0.021$ $\mathrm{mmol})$ in dry chloroform/dichloromethane $(4 / 1,3 \mathrm{~mL})$ at $-45{ }^{0} \mathrm{C}$ as internally monitored. The 
reaction was monitored very one minute interval for $40 \mathrm{~min}$ and then incrementally thereafter until complete conversion.

$\mathrm{k}_{\mathrm{obs}}=0.4428 \mathrm{M}^{-1} \min ^{-1}$

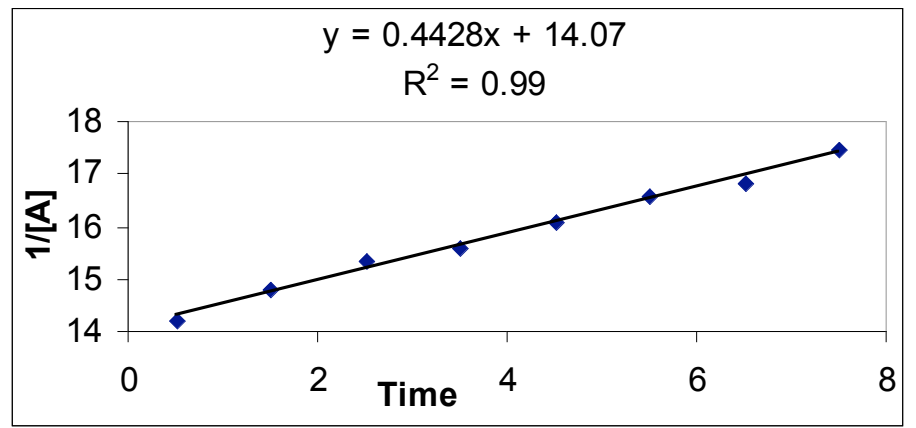

\begin{tabular}{|crrr|}
\hline $\begin{array}{c}\text { Time } \\
(\mathrm{min})\end{array}$ & {$[\mathrm{RCHO}]$} & conversion & $1 /[\mathrm{RCHO}]$ \\
0.0 & 0.0617 & 0.00 & 16.21 \\
0.5 & 0.0705 & 1.40 & 14.19 \\
1.5 & 0.0677 & 5.25 & 14.77 \\
2.5 & 0.0652 & 8.71 & 15.33 \\
3.5 & 0.0641 & 10.27 & 15.59 \\
4.5 & 0.0622 & 13.02 & 16.09 \\
5.5 & 0.0604 & 15.50 & 16.56 \\
6.5 & 0.0594 & 16.83 & 16.82 \\
7.5 & 0.0574 & 19.75 & 17.44 \\
8.5 & 0.0566 & 20.83 & 17.67 \\
9.5 & 0.0542 & 24.11 & 18.44 \\
10.5 & 0.0553 & 22.63 & 18.09 \\
11.5 & 0.0524 & 26.71 & 19.09 \\
12.5 & 0.0515 & 27.99 & 19.43 \\
13.5 & 0.0499 & 30.22 & 20.05 \\
14.5 & 0.0506 & 29.20 & 19.76 \\
15.5 & 0.0497 & 30.46 & 20.12 \\
16.5 & 0.0476 & 33.35 & 20.99 \\
17.5 & 0.0472 & 33.96 & 21.19 \\
18.5 & 0.0461 & 35.53 & 21.70 \\
19.5 & 0.0456 & 36.23 & 21.94 \\
20.5 & 0.0449 & 37.17 & 22.27 \\
21.5 & 0.0442 & 38.16 & 22.63 \\
\hline & & & \\
\hline
\end{tabular}




\begin{tabular}{|llll|}
\hline 22.5 & 0.0439 & 38.52 & 22.76 \\
23.5 & 0.0432 & 39.58 & 23.16 \\
24.5 & 0.0421 & 41.04 & 23.73 \\
25.5 & 0.0412 & 42.30 & 24.25 \\
26.5 & 0.0397 & 44.46 & 25.19 \\
27.5 & 0.0406 & 43.19 & 24.63 \\
28.5 & 0.0389 & 45.53 & 25.69 \\
29.5 & 0.0389 & 45.61 & 25.73 \\
30.5 & 0.0375 & 47.55 & 26.68 \\
31.5 & 0.0366 & 48.80 & 27.33 \\
32.5 & 0.0374 & 47.64 & 26.72 \\
33.5 & 0.0375 & 47.56 & 26.68 \\
34.5 & 0.0360 & 49.61 & 27.77 \\
35.5 & 0.0363 & 49.19 & 27.54 \\
36.5 & 0.0366 & 48.77 & 27.31 \\
37.5 & 0.0360 & 49.64 & 27.78 \\
38.5 & 0.0347 & 51.49 & 28.84 \\
39.5 & 0.0351 & 50.89 & 28.49 \\
40.5 & 0.0349 & 51.13 & 28.63 \\
\hline
\end{tabular}

Temperature: $-45^{\circ} \mathrm{C}$, run 2

Following General Procedure III, enolate $1(31.3 \mu \mathrm{L}, 0.21 \mathrm{mmol})$ was added to the solution of aldehyde $2 \mathbf{d}(41.2 \mathrm{mg}, 0.21 \mathrm{~mol})$ and the dimeric catalyst $(R, R)-\mathbf{3}(17.7 \mathrm{mg}, 0.021$ $\mathrm{mmol})$ in dry chloroform/dichloromethane $(4 / 1,3 \mathrm{~mL})$ at $-45{ }^{0} \mathrm{C}$ as internally monitored. The reaction was monitored very one minute interval for $40 \mathrm{~min}$ and then incrementally thereafter until complete conversion. 
$\mathrm{k}_{\mathrm{obs}}=0.4216 \mathrm{M}^{-1} \min ^{-1}$

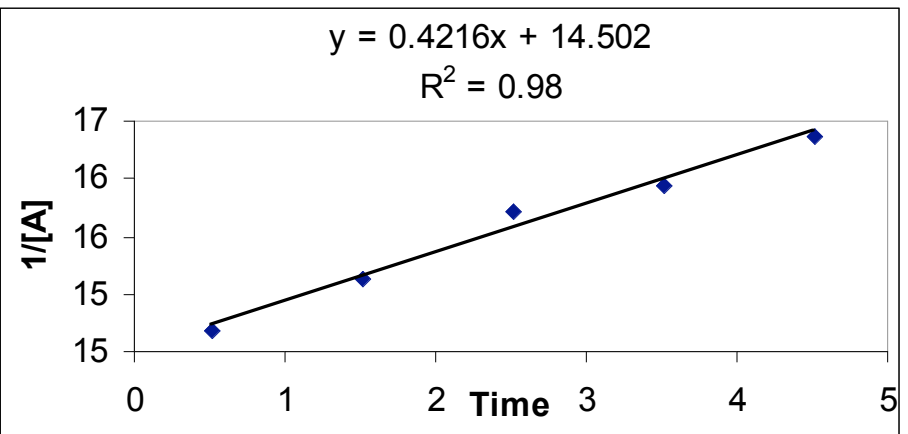

\begin{tabular}{|c|c|c|c|}
\hline $\begin{array}{l}\text { Time } \\
\text { (min) }\end{array}$ & [RCHO] & conversion & $1 /[\mathrm{RCHO}]$ \\
\hline 0.0 & 0.0616 & 0.00 & 16.23 \\
\hline 0.5 & 0.0682 & 1.45 & 14.67 \\
\hline 1.5 & 0.0661 & 4.37 & 15.12 \\
\hline 2.5 & 0.0636 & 8.00 & 15.72 \\
\hline 3.5 & 0.0627 & 9.33 & 15.94 \\
\hline 4.5 & 0.0611 & 11.65 & 16.37 \\
\hline 5.5 & 0.0598 & 13.52 & 16.72 \\
\hline 6.5 & 0.0587 & 15.20 & 17.05 \\
\hline 7.5 & 0.0575 & 16.88 & 17.39 \\
\hline 8.5 & 0.0562 & 18.78 & 17.80 \\
\hline 9.5 & 0.0552 & 20.24 & 18.13 \\
\hline 10.5 & 0.0531 & 23.25 & 18.84 \\
\hline 11.5 & 0.0534 & 22.83 & 18.73 \\
\hline 12.5 & 0.0506 & 26.90 & 19.78 \\
\hline 13.5 & 0.0502 & 27.36 & 19.90 \\
\hline 14.5 & 0.0503 & 27.27 & 19.88 \\
\hline 15.5 & 0.0493 & 28.71 & 20.28 \\
\hline 16.5 & 0.0475 & 31.27 & 21.04 \\
\hline 17.5 & 0.0471 & 31.96 & 21.25 \\
\hline 18.5 & 0.0459 & 33.65 & 21.79 \\
\hline 19.5 & 0.0458 & 33.83 & 21.85 \\
\hline 20.5 & 0.0455 & 34.20 & 21.97 \\
\hline 21.5 & 0.0444 & 35.81 & 22.53 \\
\hline 22.5 & 0.0446 & 35.45 & 22.40 \\
\hline 23.5 & 0.0429 & 37.95 & 23.30 \\
\hline 24.5 & 0.0424 & 38.65 & 23.57 \\
\hline 25.5 & 0.0425 & 38.61 & 23.55 \\
\hline 26.5 & 0.0416 & 39.91 & 24.06 \\
\hline 27.5 & 0.0400 & 42.11 & 24.97 \\
\hline 28.5 & 0.0396 & 42.69 & 25.23 \\
\hline 29.5 & 0.0397 & 42.65 & 25.21 \\
\hline
\end{tabular}




\begin{tabular}{|llll|}
\hline 30.5 & 0.0391 & 43.46 & 25.57 \\
31.5 & 0.0394 & 43.06 & 25.39 \\
32.5 & 0.0388 & 43.97 & 25.80 \\
33.5 & 0.0381 & 44.97 & 26.27 \\
34.5 & 0.0368 & 46.86 & 27.21 \\
35.5 & 0.0371 & 46.31 & 26.93 \\
36.5 & 0.0369 & 46.71 & 27.13 \\
37.5 & 0.0360 & 47.94 & 27.77 \\
38.5 & 0.0364 & 47.35 & 27.46 \\
39.5 & 0.0361 & 47.81 & 27.70 \\
40.5 & 0.0350 & 49.44 & 28.60 \\
\hline
\end{tabular}

\section{Temperature: $-52{ }^{\circ} \mathrm{C}$, run 1}

Following General Procedure III, enolate $1(31.3 \mu \mathrm{L}, 0.21 \mathrm{mmol})$ was added to the solution of aldehyde $2 \mathbf{d}(41.2 \mathrm{mg}, 0.21 \mathrm{~mol})$ and the dimeric catalyst $(R, R)-3(17.7 \mathrm{mg}, 0.021$ $\mathrm{mmol})$ in dry chloroform/dichloromethane $(4 / 1,3 \mathrm{~mL})$ at $-52{ }^{0} \mathrm{C}$ as internally monitored. The reaction was monitored very one minute interval for $40 \mathrm{~min}$ and then incrementally thereafter until complete conversion.

$\mathrm{k}_{\mathrm{obs}}=0.3911 \mathrm{M}^{-1} \min ^{-1}$

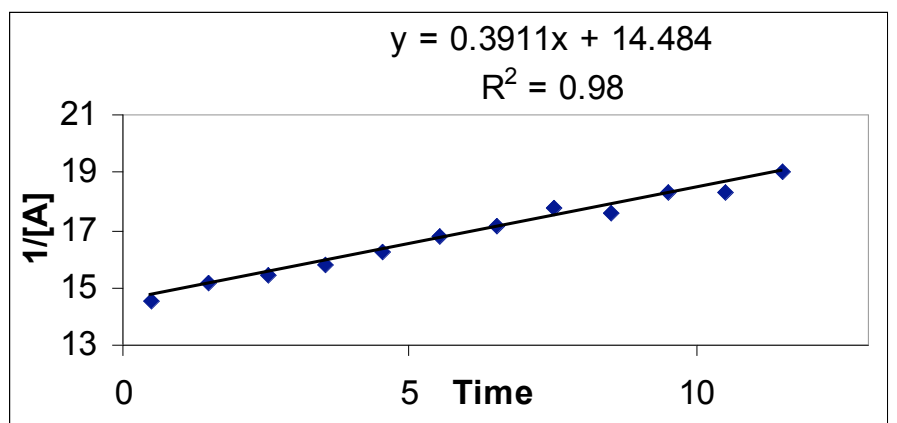

\begin{tabular}{|crrc|}
\hline $\begin{array}{c}\text { Time } \\
(\min )\end{array}$ & {$[\mathrm{RCHO}]$} & conversion & $1 /[\mathrm{RCHO}]$ \\
0.0 & 0.0617 & 0.00 & 16.21 \\
0.5 & 0.0689 & 1.44 & 14.51 \\
1.5 & 0.0659 & 5.79 & 15.17 \\
2.5 & 0.0649 & 7.25 & 15.41 \\
3.5 & 0.0635 & 9.32 & 15.75 \\
\hline
\end{tabular}




\begin{tabular}{|c|c|c|c|}
\hline 4.5 & 0.0614 & 12.23 & 16.27 \\
\hline 5.5 & 0.0596 & 14.90 & 16.78 \\
\hline 6.5 & 0.0582 & 16.89 & 17.18 \\
\hline 7.5 & 0.0562 & 19.84 & 17.80 \\
\hline 8.5 & 0.0569 & 18.85 & 17.59 \\
\hline 9.5 & 0.0546 & 22.13 & 18.32 \\
\hline 10.5 & 0.0547 & 21.95 & 18.28 \\
\hline 11.5 & 0.0527 & 24.88 & 18.98 \\
\hline 12.5 & 0.0515 & 26.59 & 19.42 \\
\hline 13.5 & 0.0516 & 26.40 & 19.37 \\
\hline 14.5 & 0.0494 & 29.52 & 20.22 \\
\hline 15.5 & 0.0487 & 30.62 & 20.54 \\
\hline 16.5 & 0.0477 & 31.98 & 20.95 \\
\hline 17.5 & 0.0469 & 33.25 & 21.34 \\
\hline 18.5 & 0.0467 & 33.51 & 21.42 \\
\hline 19.5 & 0.0452 & 35.71 & 22.15 \\
\hline 20.5 & 0.0449 & 36.06 & 22.27 \\
\hline 21.5 & 0.0430 & 38.75 & 23.23 \\
\hline 22.5 & 0.0437 & 37.81 & 22.89 \\
\hline 23.5 & 0.0427 & 39.23 & 23.41 \\
\hline 24.5 & 0.0415 & 41.02 & 24.12 \\
\hline 25.5 & 0.0408 & 41.91 & 24.48 \\
\hline 26.5 & 0.0407 & 42.14 & 24.58 \\
\hline 27.5 & 0.0405 & 42.37 & 24.67 \\
\hline 28.5 & 0.0397 & 43.52 & 25.17 \\
\hline 29.5 & 0.0398 & 43.44 & 25.13 \\
\hline 30.5 & 0.0392 & 44.28 & 25.51 \\
\hline 31.5 & 0.0378 & 46.33 & 26.47 \\
\hline 32.5 & 0.0373 & 47.07 & 26.83 \\
\hline 33.5 & 0.0381 & 45.90 & 26.26 \\
\hline 34.5 & 0.0373 & 47.04 & 26.82 \\
\hline 35.5 & 0.0360 & 48.91 & 27.79 \\
\hline 36.5 & 0.0372 & 47.22 & 26.91 \\
\hline 37.5 & 0.0354 & 49.81 & 28.28 \\
\hline 38.5 & 0.0346 & 50.97 & 28.94 \\
\hline 39.5 & 0.0354 & 49.80 & 28.27 \\
\hline 40.5 & 0.0355 & 49.65 & 28.19 \\
\hline
\end{tabular}




\section{Temperature: $-52{ }^{\circ} \mathrm{C}$, run 2}

Following General Procedure III, enolate $1(31.3 \mu \mathrm{L}, 0.21 \mathrm{mmol})$ was added to the solution of aldehyde $2 \mathbf{d}(41.2 \mathrm{mg}, 0.21 \mathrm{~mol})$ and the dimeric catalyst $(R, R)-\mathbf{3}(17.7 \mathrm{mg}, 0.021$ $\mathrm{mmol})$ in dry chloroform/dichloromethane $(4 / 1,3 \mathrm{~mL})$ at $-52{ }^{0} \mathrm{C}$ as internally monitored. The reaction was monitored very one minute interval for $40 \mathrm{~min}$ and then incrementally thereafter until complete conversion.

$$
\mathrm{k}_{\mathrm{obs}}=0.3939 \mathrm{M}^{-1} \min ^{-1}
$$

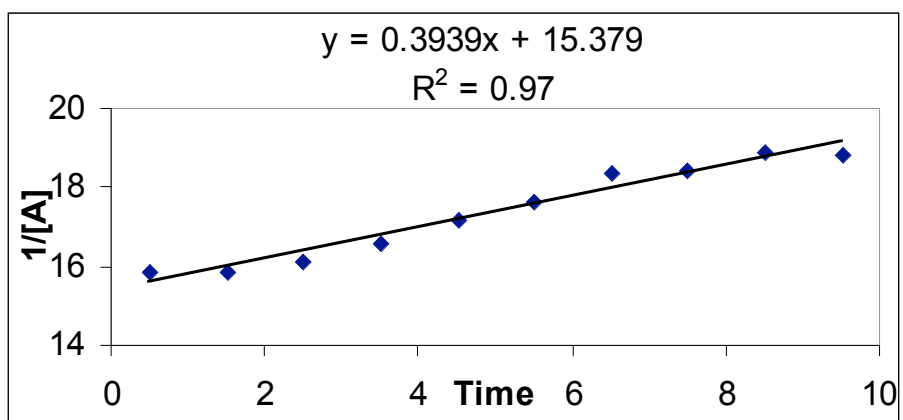

\begin{tabular}{|crrr|}
\hline $\begin{array}{c}\text { Time } \\
(\mathrm{min})\end{array}$ & {$[\mathrm{RCHO}]$} & conversion & $1 /[\mathrm{RCHO}]$ \\
0.0 & 0.0572 & 0.00 & 17.48 \\
0.5 & 0.0631 & 1.56 & 15.84 \\
1.5 & 0.0631 & 1.58 & 15.84 \\
2.5 & 0.0620 & 3.27 & 16.12 \\
3.5 & 0.0603 & 5.93 & 16.57 \\
4.5 & 0.0583 & 9.06 & 17.15 \\
5.5 & 0.0568 & 11.49 & 17.62 \\
6.5 & 0.0546 & 14.91 & 18.32 \\
7.5 & 0.0543 & 15.28 & 18.40 \\
8.5 & 0.0530 & 17.31 & 18.86 \\
9.5 & 0.0532 & 17.06 & 18.80 \\
10.5 & 0.0520 & 18.85 & 19.21 \\
11.5 & 0.0502 & 21.70 & 19.91 \\
12.5 & 0.0501 & 21.83 & 19.94 \\
13.5 & 0.0485 & 24.38 & 20.62 \\
14.5 & 0.0481 & 25.00 & 20.79 \\
15.5 & 0.0480 & 25.24 & 20.85 \\
16.5 & 0.0474 & 26.08 & 21.09 \\
\hline
\end{tabular}




\begin{tabular}{|llll|}
\hline 17.5 & 0.0464 & 27.66 & 21.55 \\
18.5 & 0.0457 & 28.81 & 21.90 \\
19.5 & 0.0445 & 30.56 & 22.45 \\
20.5 & 0.0442 & 31.09 & 22.63 \\
21.5 & 0.0444 & 30.81 & 22.53 \\
22.5 & 0.0419 & 34.66 & 23.86 \\
23.5 & 0.0425 & 33.75 & 23.54 \\
24.5 & 0.0419 & 34.60 & 23.84 \\
25.5 & 0.0405 & 36.82 & 24.68 \\
26.5 & 0.0412 & 35.73 & 24.26 \\
27.5 & 0.0405 & 36.83 & 24.68 \\
28.5 & 0.0411 & 35.88 & 24.31 \\
29.5 & 0.0399 & 37.73 & 25.04 \\
30.5 & 0.0389 & 39.40 & 25.73 \\
31.5 & 0.0391 & 39.08 & 25.59 \\
32.5 & 0.0391 & 39.10 & 25.60 \\
33.5 & 0.0389 & 39.30 & 25.69 \\
34.5 & 0.0392 & 38.89 & 25.51 \\
35.5 & 0.0380 & 40.83 & 26.35 \\
36.5 & 0.0379 & 40.91 & 26.39 \\
37.5 & 0.0370 & 42.30 & 27.02 \\
38.5 & 0.0362 & 43.51 & 27.60 \\
39.5 & 0.0360 & 43.80 & 27.74 \\
40.5 & 0.0370 & 42.26 & 27.00 \\
\hline
\end{tabular}

\section{Temperature: $-60{ }^{\circ} \mathrm{C}$, run 1}

Following General Procedure III, enolate $1(31.3 \mu \mathrm{L}, 0.21 \mathrm{mmol})$ was added to the solution of aldehyde $2 \mathbf{d}(41.2 \mathrm{mg}, 0.21 \mathrm{~mol})$ and the dimeric catalyst $(R, R)-\mathbf{3}(17.7 \mathrm{mg}, 0.021$ mmol) in dry chloroform/dichloromethane $(4 / 1,3 \mathrm{~mL})$ at $-60{ }^{\circ} \mathrm{C}$ as internally monitored. The reaction was monitored very one minute interval for $40 \mathrm{~min}$ and then incrementally thereafter until complete conversion. 
$\mathrm{k}_{\mathrm{obs}}=0.3534 \mathrm{M}^{-1} \min ^{-1}$

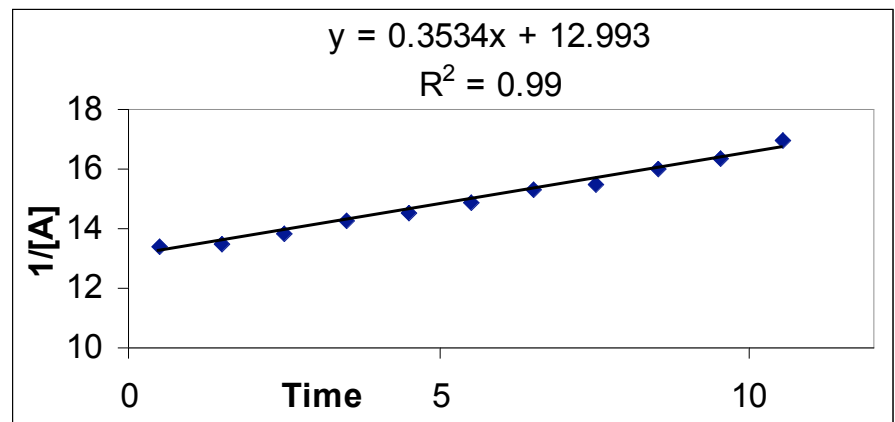

\begin{tabular}{|c|c|c|c|}
\hline $\begin{array}{l}\text { Time } \\
\text { (min) }\end{array}$ & {$[\mathrm{RCHO}]$} & conversion & $1 /[\mathrm{RCHO}]$ \\
\hline 0.0 & 0.0659 & 0.00 & 15.17 \\
\hline 0.5 & 0.0746 & 1.54 & 13.41 \\
\hline 1.5 & 0.0741 & 2.17 & 13.50 \\
\hline 2.5 & 0.0724 & 4.45 & 13.82 \\
\hline 3.5 & 0.0703 & 7.20 & 14.23 \\
\hline 4.5 & 0.0689 & 9.04 & 14.52 \\
\hline 5.5 & 0.0673 & 11.10 & 14.85 \\
\hline 6.5 & 0.0653 & 13.76 & 15.31 \\
\hline 7.5 & 0.0648 & 14.49 & 15.44 \\
\hline 8.5 & 0.0625 & 17.48 & 16.00 \\
\hline 9.5 & 0.0611 & 19.31 & 16.36 \\
\hline 10.5 & 0.0590 & 22.05 & 16.94 \\
\hline 11.5 & 0.0592 & 21.87 & 16.90 \\
\hline 12.5 & 0.0573 & 24.36 & 17.45 \\
\hline 13.5 & 0.0552 & 27.18 & 18.13 \\
\hline 14.5 & 0.0556 & 26.65 & 18.00 \\
\hline 15.5 & 0.0548 & 27.68 & 18.26 \\
\hline 16.5 & 0.0524 & 30.83 & 19.09 \\
\hline 17.5 & 0.0510 & 32.62 & 19.59 \\
\hline 18.5 & 0.0494 & 34.76 & 20.24 \\
\hline 19.5 & 0.0487 & 35.76 & 20.55 \\
\hline 20.5 & 0.0481 & 36.53 & 20.80 \\
\hline 21.5 & 0.0474 & 37.44 & 21.11 \\
\hline 22.5 & 0.0444 & 41.36 & 22.52 \\
\hline 23.5 & 0.0449 & 40.74 & 22.28 \\
\hline 24.5 & 0.0448 & 40.83 & 22.31 \\
\hline 25.5 & 0.0421 & 44.38 & 23.74 \\
\hline 26.5 & 0.0415 & 45.19 & 24.09 \\
\hline 27.5 & 0.0411 & 45.73 & 24.33 \\
\hline
\end{tabular}




\begin{tabular}{|llll|}
\hline 28.5 & 0.0403 & 46.80 & 24.82 \\
29.5 & 0.0378 & 50.10 & 26.46 \\
30.5 & 0.0363 & 52.09 & 27.56 \\
31.5 & 0.0370 & 51.13 & 27.02 \\
32.5 & 0.0341 & 55.04 & 29.36 \\
33.5 & 0.0366 & 51.64 & 27.30 \\
34.5 & 0.0326 & 57.02 & 30.72 \\
35.5 & 0.0346 & 54.35 & 28.92 \\
36.5 & 0.0324 & 57.17 & 30.83 \\
37.5 & 0.0322 & 57.53 & 31.09 \\
38.5 & 0.0326 & 57.00 & 30.70 \\
39.5 & 0.0322 & 57.47 & 31.04 \\
40.5 & 0.0318 & 58.05 & 31.47 \\
\hline
\end{tabular}

\section{Temperature: $-60{ }^{\circ} \mathrm{C}$, run 2}

Following General Procedure III, enolate $1(31.3 \mu \mathrm{L}, 0.21 \mathrm{mmol})$ was added to the solution of aldehyde $2 \mathbf{d}(41.2 \mathrm{mg}, 0.21 \mathrm{~mol})$ and the dimeric catalyst $(R, R)-\mathbf{3}(17.7 \mathrm{mg}, 0.021$ $\mathrm{mmol})$ in dry chloroform/dichloromethane $(4 / 1,3 \mathrm{~mL})$ at $-60{ }^{0} \mathrm{C}$ as internally monitored. The reaction was monitored very one minute interval for $40 \mathrm{~min}$ and then incrementally thereafter until complete conversion.

$\mathrm{k}_{\mathrm{obs}}=0.3656 \mathrm{M}^{-1} \min ^{-1}$

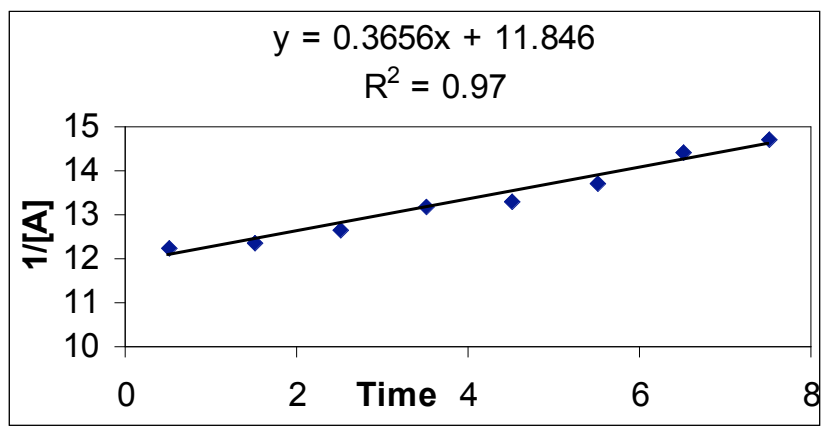

\begin{tabular}{|crcc|}
\hline $\begin{array}{c}\text { Time } \\
(\min )\end{array}$ & {$[\mathrm{RCHO}]$} & conversion & $1 /[\mathrm{RCHO}]$ \\
0.0 & 0.0716 & 0.00 & 13.97 \\
0.5 & 0.0816 & 1.21 & 12.25 \\
1.5 & 0.0808 & 2.18 & 12.37 \\
\hline
\end{tabular}




\begin{tabular}{|c|c|c|c|}
\hline 2.5 & 0.0792 & 4.20 & 12.63 \\
\hline 3.5 & 0.0760 & 8.07 & 13.16 \\
\hline 4.5 & 0.0752 & 8.98 & 13.30 \\
\hline 5.5 & 0.0730 & 11.60 & 13.69 \\
\hline 6.5 & 0.0695 & 15.93 & 14.40 \\
\hline 7.5 & 0.0679 & 17.79 & 14.72 \\
\hline 8.5 & 0.0652 & 21.08 & 15.33 \\
\hline 9.5 & 0.0659 & 20.30 & 15.18 \\
\hline 10.5 & 0.0625 & 24.38 & 16.00 \\
\hline 11.5 & 0.0616 & 25.40 & 16.22 \\
\hline 12.5 & 0.0585 & 29.21 & 17.10 \\
\hline 13.5 & 0.0591 & 28.51 & 16.93 \\
\hline 14.5 & 0.0563 & 31.91 & 17.77 \\
\hline 15.5 & 0.0537 & 34.96 & 18.61 \\
\hline 16.5 & 0.0523 & 36.69 & 19.12 \\
\hline 17.5 & 0.0520 & 37.13 & 19.25 \\
\hline 18.5 & 0.0503 & 39.17 & 19.89 \\
\hline 19.5 & 0.0499 & 39.63 & 20.05 \\
\hline 20.5 & 0.0473 & 42.73 & 21.13 \\
\hline 21.5 & 0.0457 & 44.71 & 21.89 \\
\hline 22.5 & 0.0451 & 45.47 & 22.19 \\
\hline 23.5 & 0.0437 & 47.11 & 22.88 \\
\hline 24.5 & 0.0435 & 47.30 & 22.96 \\
\hline 25.5 & 0.0424 & 48.67 & 23.58 \\
\hline 26.5 & 0.0417 & 49.58 & 24.00 \\
\hline 27.5 & 0.0403 & 51.28 & 24.84 \\
\hline 28.5 & 0.0381 & 53.85 & 26.22 \\
\hline 29.5 & 0.0385 & 53.41 & 25.98 \\
\hline 30.5 & 0.0382 & 53.79 & 26.19 \\
\hline 31.5 & 0.0375 & 54.61 & 26.66 \\
\hline 32.5 & 0.0363 & 56.02 & 27.52 \\
\hline 33.5 & 0.0347 & 58.01 & 28.82 \\
\hline 34.5 & 0.0348 & 57.84 & 28.71 \\
\hline 35.5 & 0.0346 & 58.09 & 28.87 \\
\hline 36.5 & 0.0327 & 60.49 & 30.63 \\
\hline 37.5 & 0.0325 & 60.66 & 30.76 \\
\hline 38.5 & 0.0313 & 62.14 & 31.96 \\
\hline 39.5 & 0.0301 & 63.60 & 33.25 \\
\hline 40.5 & 0.0309 & 62.66 & 32.41 \\
\hline
\end{tabular}




\section{Temperature: $-72{ }^{\circ} \mathrm{C}$, run 1}

Following General Procedure III, enolate $1(31.3 \mu \mathrm{L}, 0.21 \mathrm{mmol})$ was added to the solution of aldehyde $\mathbf{2 d}(41.2 \mathrm{mg}, 0.21 \mathrm{~mol})$ and the dimeric catalyst $(R, R)-\mathbf{3}(17.7 \mathrm{mg}, 0.021$ $\mathrm{mmol})$ in dry chloroform/dichloromethane $(4 / 1,3 \mathrm{~mL})$ at $-72{ }^{\circ} \mathrm{C}$ as internally monitored. The reaction was monitored very one minute interval for $40 \mathrm{~min}$ and then incrementally thereafter until complete conversion.

$$
\mathrm{k}_{\mathrm{obs}}=0.2505 \mathrm{M}^{-1} \min ^{-1}
$$

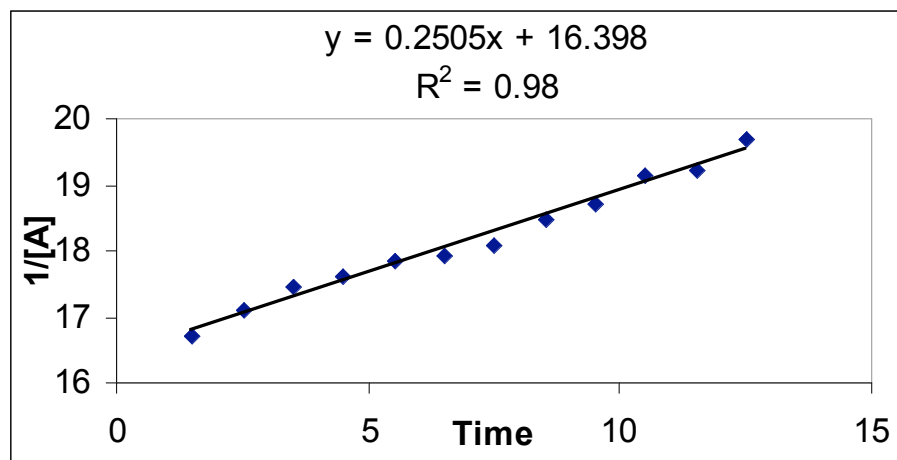

\begin{tabular}{|crcc|}
\hline $\begin{array}{c}\text { Time } \\
(\mathrm{min})\end{array}$ & {$[\mathrm{RCHO}]$} & conversion & $1 /[\mathrm{RCHO}]$ \\
0.0 & 0.0550 & 0.00 & 18.18 \\
0.5 & 0.0598 & 1.64 & 16.73 \\
1.5 & 0.0599 & 1.43 & 16.69 \\
2.5 & 0.0585 & 3.69 & 17.08 \\
3.5 & 0.0573 & 5.66 & 17.44 \\
4.5 & 0.0568 & 6.49 & 17.59 \\
5.5 & 0.0560 & 7.82 & 17.85 \\
6.5 & 0.0558 & 8.25 & 17.93 \\
7.5 & 0.0553 & 8.94 & 18.07 \\
8.5 & 0.0542 & 10.83 & 18.45 \\
9.5 & 0.0534 & 12.07 & 18.71 \\
10.5 & 0.0523 & 14.03 & 19.14 \\
11.5 & 0.0521 & 14.35 & 19.21 \\
12.5 & 0.0508 & 16.48 & 19.70 \\
13.5 & 0.0510 & 16.11 & 19.61 \\
14.5 & 0.0503 & 17.32 & 19.90 \\
15.5 & 0.0494 & 18.79 & 20.26 \\
\hline
\end{tabular}




\begin{tabular}{|llll|}
\hline 16.5 & 0.0497 & 18.27 & 20.13 \\
17.5 & 0.0492 & 19.12 & 20.34 \\
18.5 & 0.0479 & 21.16 & 20.87 \\
19.5 & 0.0482 & 20.63 & 20.73 \\
20.5 & 0.0473 & 22.20 & 21.15 \\
21.5 & 0.0474 & 22.04 & 21.11 \\
22.5 & 0.0463 & 23.83 & 21.60 \\
23.5 & 0.0470 & 22.65 & 21.27 \\
24.5 & 0.0455 & 25.18 & 21.99 \\
25.5 & 0.0441 & 27.45 & 22.68 \\
26.5 & 0.0445 & 26.83 & 22.49 \\
27.5 & 0.0447 & 26.51 & 22.39 \\
28.5 & 0.0450 & 25.98 & 22.23 \\
29.5 & 0.0438 & 27.93 & 22.83 \\
30.5 & 0.0436 & 28.34 & 22.96 \\
31.5 & 0.0433 & 28.80 & 23.11 \\
32.5 & 0.0423 & 30.34 & 23.62 \\
33.5 & 0.0413 & 32.02 & 24.20 \\
34.5 & 0.0424 & 30.22 & 23.58 \\
35.5 & 0.0412 & 32.22 & 24.28 \\
36.5 & 0.0419 & 31.12 & 23.88 \\
37.5 & 0.0403 & 33.67 & 24.81 \\
38.5 & 0.0400 & 34.14 & 24.98 \\
39.5 & 0.0392 & 35.43 & 25.48 \\
40.5 & 0.0383 & 36.91 & 26.08 \\
\hline & & & \\
\hline
\end{tabular}

\section{Temperature: $-72{ }^{\circ} \mathrm{C}$, run 2}

Following General Procedure III, enolate $1(31.3 \mu \mathrm{L}, 0.21 \mathrm{mmol})$ was added to the solution of aldehyde $2 \mathbf{d}(41.2 \mathrm{mg}, 0.21 \mathrm{~mol})$ and the dimeric catalyst $(R, R)-\mathbf{3}(17.7 \mathrm{mg}, 0.021$ $\mathrm{mmol})$ in dry chloroform/dichloromethane $(4 / 1,3 \mathrm{~mL})$ at $-72{ }^{0} \mathrm{C}$ as internally monitored. The reaction was monitored very one minute interval for $40 \mathrm{~min}$ and then incrementally thereafter until complete conversion. 
$\mathrm{k}_{\mathrm{obs}}=0.2549 \mathrm{M}^{-1} \min ^{-1}$

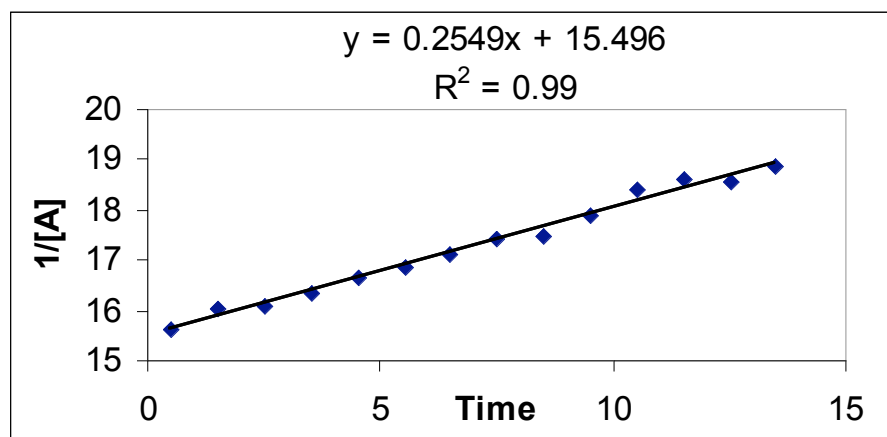

\begin{tabular}{|c|c|c|c|}
\hline $\begin{array}{l}\text { Time } \\
\text { (min) }\end{array}$ & [RCHO] & conversion & $1 /[\mathrm{RCHO}]$ \\
\hline 0.0 & 0.0545 & 0.00 & 18.33 \\
\hline 0.5 & 0.0640 & 1.53 & 15.62 \\
\hline 1.5 & 0.0625 & 3.95 & 16.01 \\
\hline 2.5 & 0.0621 & 4.44 & 16.09 \\
\hline 3.5 & 0.0612 & 5.87 & 16.34 \\
\hline 4.5 & 0.0600 & 7.73 & 16.67 \\
\hline 5.5 & 0.0593 & 8.80 & 16.87 \\
\hline 6.5 & 0.0584 & 10.10 & 17.11 \\
\hline 7.5 & 0.0574 & 11.65 & 17.42 \\
\hline 8.5 & 0.0572 & 12.06 & 17.50 \\
\hline 9.5 & 0.0560 & 13.89 & 17.87 \\
\hline 10.5 & 0.0543 & 16.41 & 18.41 \\
\hline 11.5 & 0.0537 & 17.38 & 18.63 \\
\hline 12.5 & 0.0538 & 17.16 & 18.58 \\
\hline 13.5 & 0.0530 & 18.38 & 18.86 \\
\hline 14.5 & 0.0530 & 18.37 & 18.86 \\
\hline 15.5 & 0.0533 & 17.90 & 18.75 \\
\hline 16.5 & 0.0530 & 18.38 & 18.86 \\
\hline 17.5 & 0.0521 & 19.74 & 19.18 \\
\hline 18.5 & 0.0505 & 22.28 & 19.81 \\
\hline 19.5 & 0.0498 & 23.34 & 20.09 \\
\hline 20.5 & 0.0489 & 24.69 & 20.45 \\
\hline 21.5 & 0.0480 & 26.11 & 20.85 \\
\hline 22.5 & 0.0476 & 26.73 & 21.03 \\
\hline 23.5 & 0.0479 & 26.15 & 20.86 \\
\hline 24.5 & 0.0466 & 28.22 & 21.47 \\
\hline 25.5 & 0.0462 & 28.79 & 21.64 \\
\hline 26.5 & 0.0467 & 28.11 & 21.43 \\
\hline 27.5 & 0.0459 & 29.22 & 21.77 \\
\hline 28.5 & 0.0469 & 27.79 & 21.34 \\
\hline 29.5 & 0.0443 & 31.78 & 22.60 \\
\hline
\end{tabular}




\begin{tabular}{|llll|}
\hline 30.5 & 0.0460 & 29.11 & 21.74 \\
31.5 & 0.0450 & 30.67 & 22.23 \\
32.5 & 0.0454 & 29.96 & 22.00 \\
33.5 & 0.0436 & 32.73 & 22.91 \\
34.5 & 0.0447 & 31.17 & 22.39 \\
35.5 & 0.0434 & 33.11 & 23.05 \\
36.5 & 0.0441 & 32.01 & 22.67 \\
37.5 & 0.0417 & 35.65 & 23.97 \\
38.5 & 0.0432 & 33.37 & 23.14 \\
39.5 & 0.0416 & 35.78 & 24.01 \\
40.5 & 0.0411 & 36.59 & 24.32 \\
\hline
\end{tabular}

\section{Arrhenius plot for $\mathbf{2 d}$}

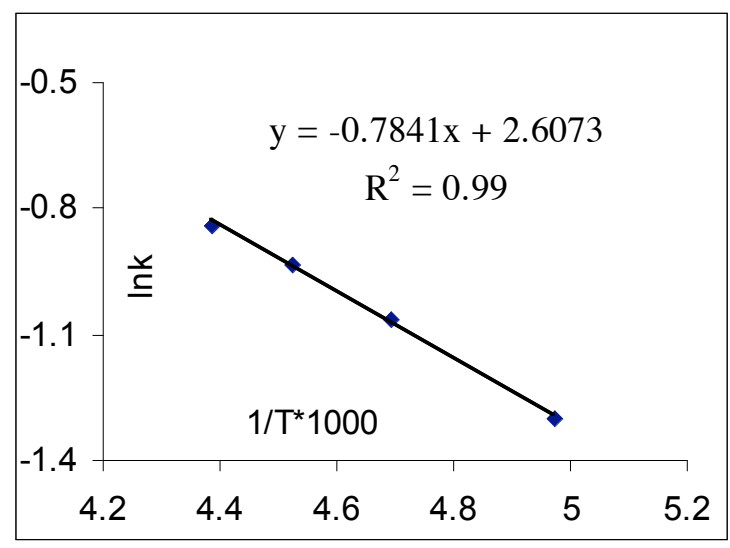

\section{Calculation of activation parameters at $201 \mathrm{~K}^{4}$}

slope $=-0.7841 \quad\left(\right.$ from graph of $\mathrm{k}_{\mathrm{obs}}$ vs $1 / \mathrm{T}$, aldehyde $\left.2 \mathbf{d}\right)$

$\mathrm{k}_{\mathrm{obs}}=\mathrm{A} \mathrm{e}^{-\mathrm{Ea} / \mathrm{RT}}$ where $\mathrm{R}=1.987 \mathrm{cal} / \mathrm{molK}$

$\mathrm{A}=$ preexponential factor

$$
\mathrm{T}=201 \mathrm{~K}\left(72^{\circ} \mathrm{C}\right)
$$

$\mathrm{e}^{-\mathrm{E} a / \mathrm{RT}}=$ exponential factor

slope $=-\mathrm{E}_{\mathrm{a}} / \mathrm{R} \Longrightarrow \mathrm{E}_{\mathrm{a}}=\mathrm{R} \times$ slope $=1.987 \times 0.7841=1.6 \mathrm{Kcal} / \mathrm{mol}$

$\Delta \mathrm{H}^{\ddagger}=\mathrm{E}_{\mathrm{a}}-\mathrm{RT}=1558-1.987(201)=1158.6 \mathrm{cal} / \mathrm{mol}=1.2 \mathrm{kcal} / \mathrm{mol}$

$\Delta \mathrm{S}^{\ddagger}=4.576 \log (\mathrm{A} / \mathrm{T})-49.203=-54.58 \mathrm{eu}$

$\Delta \mathrm{G}^{\ddagger}=\Delta \mathrm{H}^{\ddagger}-\mathrm{T} \Delta \mathrm{S}^{\ddagger}=1158.6-(201)(-54.58)=12109.1 \mathrm{cal} / \mathrm{mol}=12.1 \mathrm{Kcal} / \mathrm{mol}$ 


\section{Kinetic Data for $2 f$}
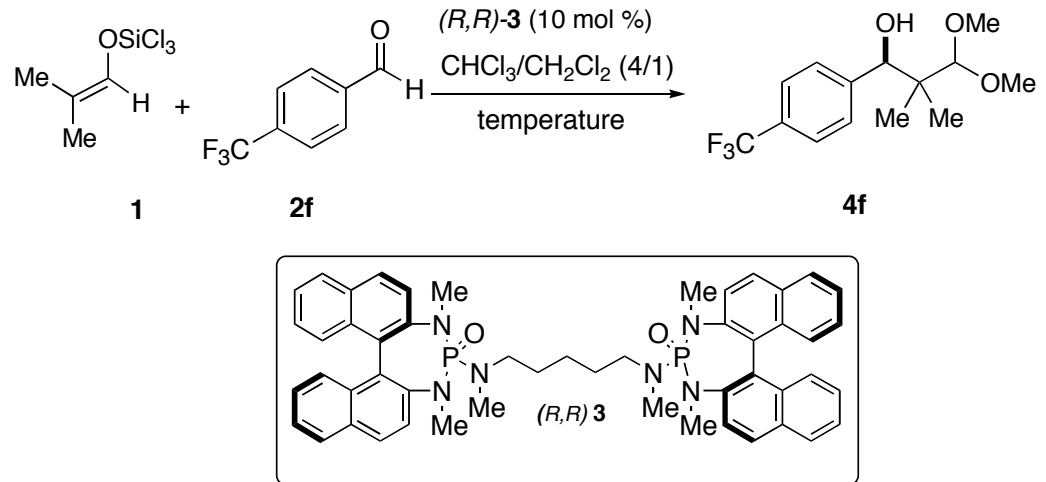

\section{Temperature: $-45^{\circ} \mathrm{C}$, run 1}

Following General Procedure III, enolate $1(14.9 \mu \mathrm{L}, 0.10 \mathrm{mmol})$ was added to the solution of aldehyde $2 \mathbf{f}(13.7 \mu \mathrm{L}, 0.10 \mathrm{~mol})$ and the dimeric catalyst $(R, R)-3(8.42 \mathrm{mg}, 0.01$ $\mathrm{mmol})$ in dry chloroform/dichloromethane $(4 / 1,3 \mathrm{~mL})$ at $-45{ }^{0} \mathrm{C}$ as internally monitored. The reaction was monitored very thirty second interval for about $9 \mathrm{~min}$.

$\mathrm{k}_{\mathrm{obs}}=22.28 \mathrm{M}^{-1} \min ^{-1}$

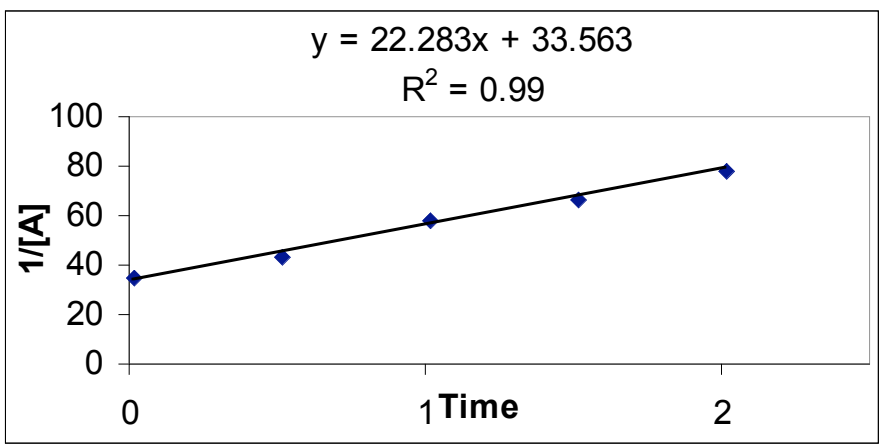

\begin{tabular}{|crcc|}
\hline $\begin{array}{c}\text { Time } \\
(\mathrm{min})\end{array}$ & {$[\mathrm{RCHO}]$} & conversion & $1 /[\mathrm{RCHO}]$ \\
0.0 & 0.0292 & 0.00 & 34.27 \\
0.5 & 0.0230 & 21.18 & 43.48 \\
1.0 & 0.0172 & 41.23 & 58.31 \\
1.5 & 0.0150 & 48.59 & 66.65 \\
2.0 & 0.0128 & 56.28 & 78.39 \\
\hline
\end{tabular}




\begin{tabular}{|llll|}
\hline 2.5 & 0.0103 & 64.86 & 97.52 \\
3.0 & 0.0104 & 64.43 & 96.34 \\
3.5 & 0.0067 & 77.08 & 149.5 \\
4.0 & 0.0063 & 78.31 & 158.0 \\
4.5 & 0.0079 & 72.87 & 126.3 \\
5.0 & 0.0067 & 76.92 & 148.5 \\
5.5 & 0.0056 & 80.83 & 178.8 \\
6.0 & 0.0056 & 80.91 & 179.5 \\
6.5 & 0.0055 & 81.12 & 181.5 \\
7.0 & 0.0051 & 82.42 & 194.9 \\
7.5 & 0.0036 & 87.66 & 277.7 \\
8.0 & 0.0044 & 84.78 & 225.2 \\
8.5 & 0.0046 & 84.16 & 216.4 \\
\hline
\end{tabular}

\section{Temperature: $-45^{\circ} \mathrm{C}$, run 2}

Following General Procedure III, enolate $1(14.9 \mu \mathrm{L}, 0.10 \mathrm{mmol})$ was added to the solution of aldehyde $2 \mathbf{f}(13.7 \mu \mathrm{L}, 0.10 \mathrm{~mol})$ and the dimeric catalyst $(R, R)-3(8.42 \mathrm{mg}, 0.01$ mmol) in dry chloroform/dichloromethane $(4 / 1,3 \mathrm{~mL})$ at $-45{ }^{0} \mathrm{C}$ as internally monitored. The reaction was monitored very thirty second interval for about 9 min.

$\mathrm{k}_{\mathrm{obs}}=22.86 \mathrm{M}^{-1} \min ^{-1}$

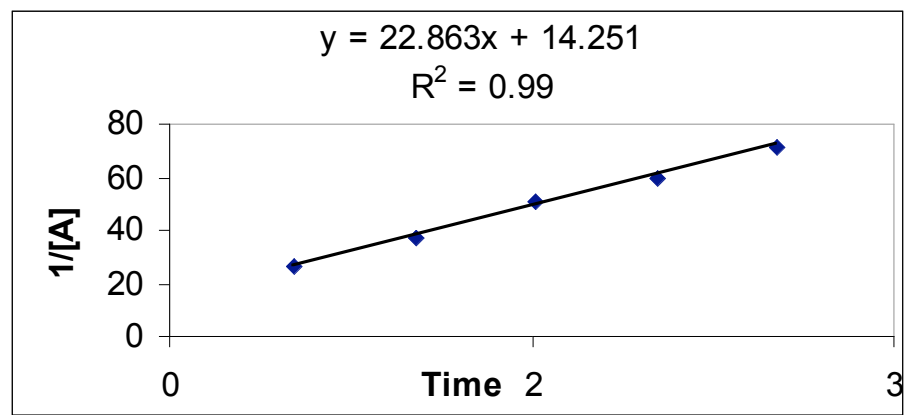

\begin{tabular}{|crcc|}
\hline $\begin{array}{c}\text { Time } \\
(\mathrm{min})\end{array}$ & {$[\mathrm{RCHO}]$} & conversion & $1 /[\mathrm{RCHO}]$ \\
0.0 & 0.0388 & 0.00 & 25.77 \\
0.5 & 0.0386 & 0.57 & 25.91 \\
1.0 & 0.0272 & 0.58 & 36.83 \\
1.5 & 0.0198 & 30.05 & 50.42 \\
2.0 & 0.0167 & 48.90 & 59.97 \\
\hline
\end{tabular}




\begin{tabular}{|llll|}
\hline 2.5 & 0.0140 & 57.04 & 71.50 \\
3.0 & 0.0108 & 63.97 & 92.69 \\
3.5 & 0.0105 & 72.20 & 95.68 \\
4.0 & 0.0098 & 73.07 & 102.1 \\
4.5 & 0.0095 & 74.76 & 105.5 \\
5.0 & 0.0083 & 75.58 & 119.9 \\
5.5 & 0.0098 & 78.51 & 102.0 \\
6.0 & 0.0081 & 74.75 & 123.7 \\
6.5 & 0.0074 & 79.17 & 134.4 \\
7.0 & 0.0078 & 80.82 & 128.7 \\
7.5 & 0.0063 & 79.98 & 159.8 \\
8.0 & 0.0067 & 83.88 & 148.5 \\
8.5 & 0.0070 & 82.65 & 143.0 \\
\hline
\end{tabular}

\section{Temperature: $-55^{\circ} \mathrm{C}$, run 1}

Following General Procedure III, enolate $1(14.9 \mu \mathrm{L}, 0.10 \mathrm{mmol})$ was added to the solution of aldehyde $2 \mathbf{f}(13.7 \mu \mathrm{L}, 0.10 \mathrm{~mol})$ and the dimeric catalyst $(R, R)-3(8.42 \mathrm{mg}, 0.01$ $\mathrm{mmol})$ in dry chloroform/dichloromethane $(4 / 1,3 \mathrm{~mL})$ at $-55{ }^{0} \mathrm{C}$ as internally monitored. The reaction was monitored very thirty second interval for about 9 min.

$\mathrm{k}_{\mathrm{obs}}=14.41 \mathrm{M}^{-1} \min ^{-1}$

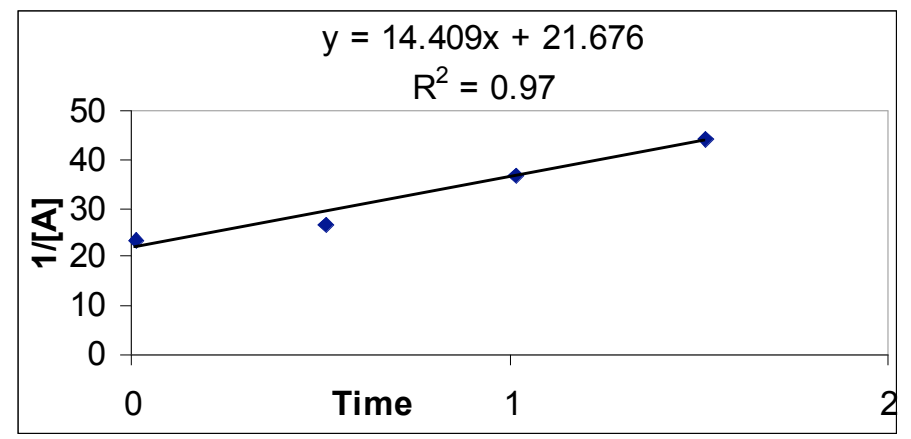

\begin{tabular}{|crcc|}
\hline $\begin{array}{c}\text { Time } \\
(\min )\end{array}$ & {$[\mathrm{RCHO}]$} & conversion & $1 /[\mathrm{RCHO}]$ \\
0.0 & 0.0427 & 0.00 & \\
0.5 & 0.0376 & 12.05 & 23.41 \\
1.0 & 0.0271 & 36.51 & 26.61 \\
1.5 & 0.0227 & 46.81 & 36.86 \\
2.0 & 0.0222 & 47.97 & 44.00 \\
2.5 & 0.0210 & 50.79 & 44.99 \\
\hline
\end{tabular}




\begin{tabular}{|llll|}
\hline 3.0 & 0.0213 & 50.24 & 47.04 \\
3.5 & 0.0203 & 52.49 & 49.27 \\
4.0 & 0.0206 & 51.75 & 48.51 \\
4.5 & 0.0206 & 51.71 & 48.47 \\
5.0 & 0.0206 & 51.73 & 48.49 \\
5.5 & 0.0187 & 56.17 & 53.40 \\
6.0 & 0.0191 & 55.22 & 52.27 \\
6.5 & 0.0200 & 53.11 & 49.92 \\
7.0 & 0.0194 & 54.53 & 51.47 \\
7.5 & 0.0204 & 52.30 & 49.07 \\
8.0 & 0.0191 & 55.29 & 52.36 \\
8.5 & 0.0176 & 58.87 & 56.92 \\
\hline
\end{tabular}

\section{Temperature: $-55{ }^{\circ} \mathrm{C}$, run 2}

Following General Procedure III, enolate $1(14.9 \mu \mathrm{L}, 0.10 \mathrm{mmol})$ was added to the solution of aldehyde $\mathbf{2 f}(13.7 \mu \mathrm{L}, 0.10 \mathrm{~mol})$ and the dimeric catalyst $(R, R)-3(8.42 \mathrm{mg}, 0.01$ $\mathrm{mmol})$ in dry chloroform/dichloromethane $(4 / 1,3 \mathrm{~mL})$ at $-55{ }^{0} \mathrm{C}$ as internally monitored. The reaction was monitored very thirty second interval for about 9 min.

$\mathrm{k}_{\mathrm{obs}}=16.52 \mathrm{M}^{-1} \min ^{-1}$

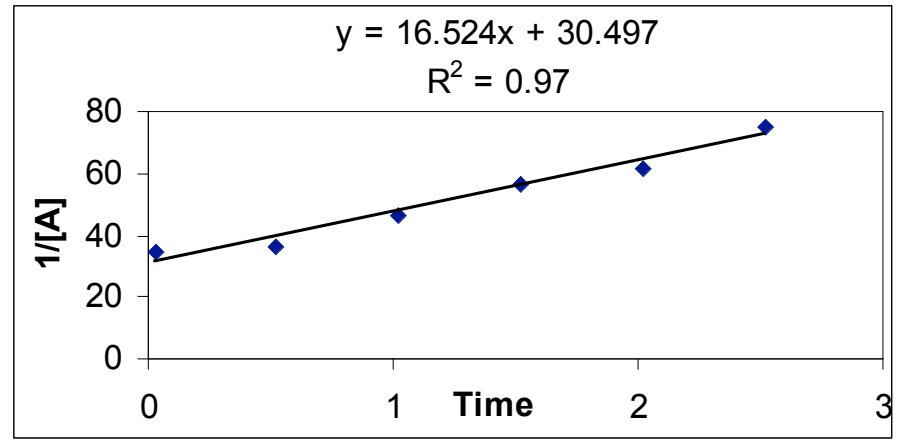

\begin{tabular}{|crcc|}
\hline $\begin{array}{c}\text { Time } \\
(\min )\end{array}$ & {$[\mathrm{RCHO}]$} & conversion & $1 /[\mathrm{RCHO}]$ \\
0.0 & 0.0292 & 0.00 & 34.30 \\
0.5 & 0.0278 & 4.78 & 36.03 \\
1.0 & 0.0217 & 25.71 & 46.18 \\
1.5 & 0.0177 & 39.28 & 56.50 \\
2.0 & 0.0163 & 43.91 & 61.16 \\
\hline
\end{tabular}




\begin{tabular}{|llll|}
\hline 2.5 & 0.0134 & 54.06 & 74.67 \\
3.0 & 0.0127 & 56.43 & 78.74 \\
3.5 & 0.0137 & 52.89 & 72.82 \\
4.0 & 0.0125 & 57.21 & 80.17 \\
4.5 & 0.0123 & 57.82 & 81.32 \\
5.0 & 0.0133 & 54.46 & 75.33 \\
5.5 & 0.0115 & 60.62 & 87.12 \\
6.0 & 0.0121 & 58.47 & 82.61 \\
6.5 & 0.0113 & 61.33 & 88.71 \\
7.0 & 0.0109 & 62.44 & 91.33 \\
7.5 & 0.0100 & 65.68 & 99.94 \\
8.0 & 0.0101 & 65.32 & 98.93 \\
8.5 & 0.0103 & 64.51 & 96.67 \\
\hline
\end{tabular}

\section{Temperature: $-65^{\circ} \mathrm{C}$, run 1}

Following General Procedure III, enolate $1(14.9 \mu \mathrm{L}, 0.10 \mathrm{mmol})$ was added to the solution of aldehyde $2 \mathbf{f}(13.7 \mu \mathrm{L}, 0.10 \mathrm{~mol})$ and the dimeric catalyst $(R, R)-3(8.42 \mathrm{mg}, 0.01$ $\mathrm{mmol})$ in dry chloroform/dichloromethane $(4 / 1,3 \mathrm{~mL})$ at $-65{ }^{0} \mathrm{C}$ as internally monitored. The reaction was monitored very thirty second interval for about 9 min.

$$
\mathrm{k}_{\mathrm{obs}}=12.49 \mathrm{M}^{-1} \min ^{-1}
$$

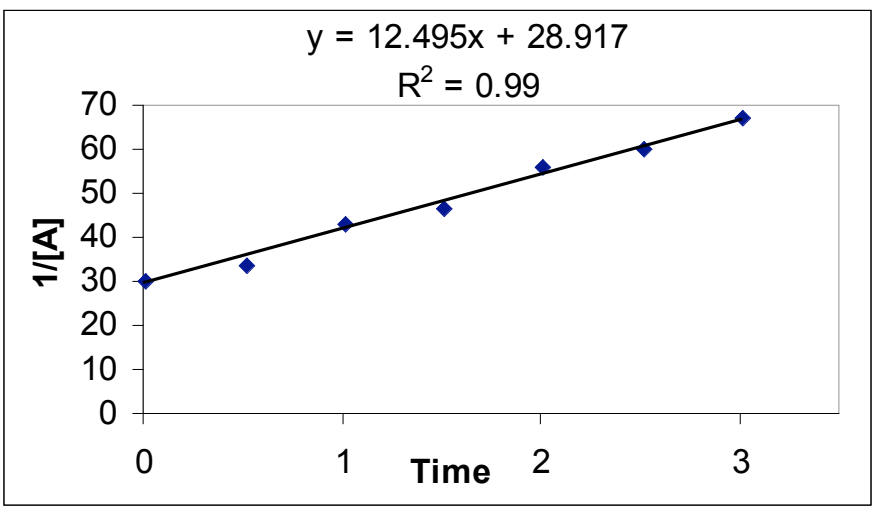

\begin{tabular}{|crcc|}
\hline $\begin{array}{c}\text { Time } \\
(\min )\end{array}$ & {$[\mathrm{RCHO}]$} & conversion & $1 /[\mathrm{RCHO}]$ \\
0.0 & 0.0333 & 0.00 & 30.02 \\
0.5 & 0.0296 & 11.14 & 33.78 \\
1.0 & 0.0233 & 30.08 & 42.94 \\
\hline
\end{tabular}




\begin{tabular}{|llll|}
\hline 1.5 & 0.0217 & 35.00 & 46.19 \\
2.0 & 0.0180 & 46.01 & 55.61 \\
2.5 & 0.0167 & 49.75 & 59.75 \\
3.0 & 0.0150 & 55.06 & 66.80 \\
3.5 & 0.0152 & 54.35 & 65.77 \\
4.0 & 0.0116 & 65.22 & 86.32 \\
4.5 & 0.0113 & 66.16 & 88.72 \\
5.0 & 0.0121 & 63.78 & 82.89 \\
5.5 & 0.0114 & 65.79 & 87.76 \\
6.0 & 0.0105 & 68.38 & 94.93 \\
6.5 & 0.0105 & 68.33 & 94.79 \\
7.0 & 0.0105 & 68.55 & 95.45 \\
7.5 & 0.0109 & 67.33 & 91.89 \\
8.0 & 0.0100 & 69.89 & 99.70 \\
8.5 & 0.0100 & 70.01 & 100.1 \\
\hline
\end{tabular}

\section{Temperature: $-65^{\circ} \mathrm{C}$, run 2}

Following General Procedure III, enolate $1(14.9 \mu \mathrm{L}, 0.10 \mathrm{mmol})$ was added to the solution of aldehyde $2 \mathbf{f}(13.7 \mu \mathrm{L}, 0.10 \mathrm{~mol})$ and the dimeric catalyst $(R, R)-3(8.42 \mathrm{mg}, 0.01$ $\mathrm{mmol})$ in dry chloroform/dichloromethane $(4 / 1,3 \mathrm{~mL})$ at $-65{ }^{0} \mathrm{C}$ as internally monitored. The reaction was monitored very thirty second interval for about 9 min.

$\mathrm{k}_{\mathrm{obs}}=13.34 \mathrm{M}^{-1} \min ^{-1}$

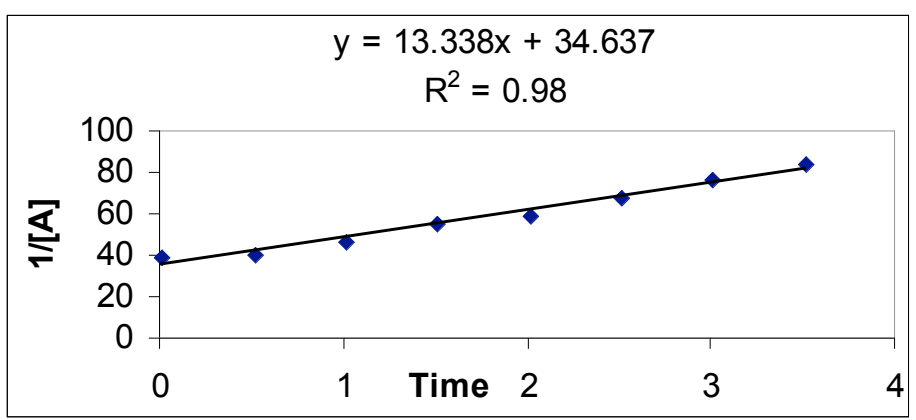

\begin{tabular}{|cccc|}
\hline $\begin{array}{c}\text { Time } \\
(\min )\end{array}$ & {$[\mathrm{RCHO}]$} & conversion & $1 /[\mathrm{RCHO}]$ \\
0.0 & 0.0259 & 0.00 & 38.56 \\
0.5 & 0.0250 & 3.56 & 39.98 \\
1.0 & 0.0214 & 17.49 & 46.73 \\
\hline
\end{tabular}




\begin{tabular}{|llll|}
\hline 1.5 & 0.0181 & 30.14 & 55.19 \\
2.0 & 0.0171 & 34.03 & 58.45 \\
2.5 & 0.0149 & 42.56 & 67.13 \\
3.0 & 0.0132 & 49.07 & 75.70 \\
3.5 & 0.0119 & 54.02 & 83.87 \\
4.0 & 0.0125 & 51.72 & 79.86 \\
4.5 & 0.0118 & 54.63 & 84.98 \\
5.0 & 0.0118 & 54.64 & 85.01 \\
5.5 & 0.0104 & 59.95 & 96.28 \\
6.0 & 0.0102 & 60.80 & 98.37 \\
6.5 & 0.0104 & 59.88 & 96.11 \\
7.0 & 0.0103 & 60.34 & 97.21 \\
7.5 & 0.0095 & 63.40 & 105.3 \\
8.0 & 0.0082 & 68.39 & 122.0 \\
8.5 & 0.0087 & 66.44 & 114.9 \\
\hline
\end{tabular}

\section{Temperature: $-73^{\circ} \mathrm{C}$, run 1}

Following General Procedure III, enolate $1(14.9 \mu \mathrm{L}, 0.10 \mathrm{mmol})$ was added to the solution of aldehyde $2 \mathbf{f}(13.7 \mu \mathrm{L}, 0.10 \mathrm{~mol})$ and the dimeric catalyst $(R, R)-3(8.42 \mathrm{mg}, 0.01$ $\mathrm{mmol})$ in dry chloroform/dichloromethane $(4 / 1,3 \mathrm{~mL})$ at $-73{ }^{0} \mathrm{C}$ as internally monitored. The reaction was monitored very thirty second interval for about $9 \mathrm{~min}$.

$\mathrm{k}_{\mathrm{obs}}=7.347 \mathrm{M}^{-1} \min ^{-1}$

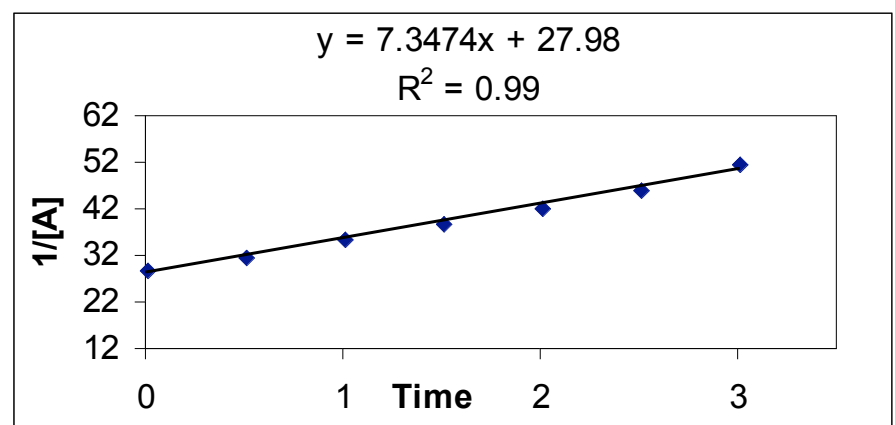

\begin{tabular}{|crcc|}
\hline $\begin{array}{c}\text { Time } \\
(\min )\end{array}$ & {$[\mathrm{RCHO}]$} & conversion & $1 /[\mathrm{RCHO}]$ \\
0.0 & 0.0347 & 0.00 & 28.83 \\
0.5 & 0.0316 & 8.81 & 31.60 \\
\hline
\end{tabular}




\begin{tabular}{|llll|}
\hline 1.0 & 0.0284 & 18.25 & 35.25 \\
1.5 & 0.0258 & 25.77 & 38.82 \\
2.0 & 0.0237 & 31.66 & 42.17 \\
2.5 & 0.0218 & 37.24 & 45.92 \\
3.0 & 0.0195 & 43.79 & 51.27 \\
3.5 & 0.0193 & 44.31 & 51.75 \\
4.0 & 0.0174 & 49.92 & 57.54 \\
4.5 & 0.0177 & 48.92 & 56.41 \\
5.0 & 0.0164 & 52.63 & 60.83 \\
5.5 & 0.0157 & 54.77 & 63.72 \\
6.0 & 0.0143 & 58.84 & 70.01 \\
6.5 & 0.0132 & 61.92 & 75.68 \\
7.0 & 0.0136 & 60.75 & 73.42 \\
7.5 & 0.0128 & 63.23 & 78.37 \\
8.0 & 0.0129 & 62.73 & 77.32 \\
8.5 & 0.0116 & 66.47 & 85.96 \\
\hline
\end{tabular}

\section{Temperature: $-73^{\circ} \mathrm{C}$, run 2}

Following General Procedure III, enolate $1(14.9 \mu \mathrm{L}, 0.10 \mathrm{mmol})$ was added to the solution of aldehyde $2 \mathbf{f}(13.7 \mu \mathrm{L}, 0.10 \mathrm{~mol})$ and the dimeric catalyst $(R, R)-3(8.42 \mathrm{mg}, 0.01$ $\mathrm{mmol})$ in dry chloroform/dichloromethane $(4 / 1,3 \mathrm{~mL})$ at $-73{ }^{0} \mathrm{C}$ as internally monitored. The reaction was monitored very thirty second interval for about $9 \mathrm{~min}$.

$\mathrm{k}_{\mathrm{obs}}=7.366 \mathrm{M}^{-1} \mathrm{~min}^{-1}$

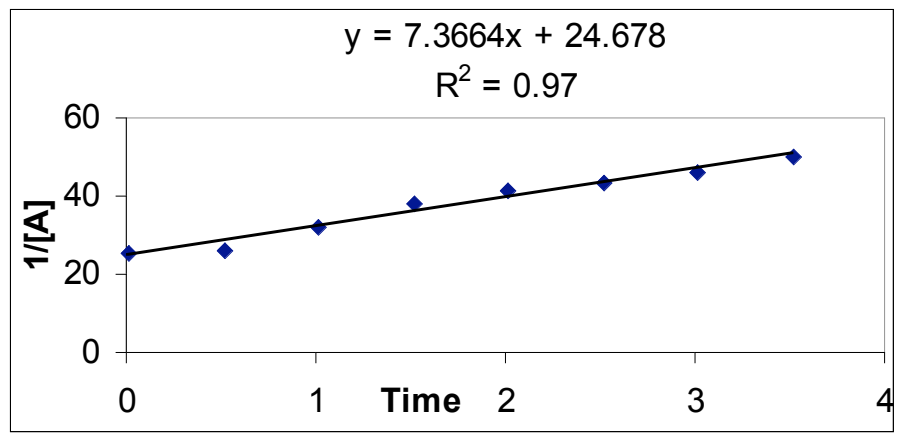




\begin{tabular}{|crrr|}
\hline $\begin{array}{c}\text { Time } \\
(\mathrm{min})\end{array}$ & {$[\mathrm{RCHO}]$} & conversion & $1 /[\mathrm{RCHO}]$ \\
0.0 & 0.0394 & 0.00 & 25.36 \\
0.5 & 0.0386 & 9.07 & 25.91 \\
1.0 & 0.0315 & 25.74 & 31.73 \\
1.5 & 0.0262 & 38.31 & 38.20 \\
2.0 & 0.0242 & 42.88 & 41.25 \\
2.5 & 0.0232 & 45.36 & 43.13 \\
3.0 & 0.0216 & 48.99 & 46.19 \\
3.5 & 0.0201 & 52.64 & 49.75 \\
4.0 & 0.0208 & 51.06 & 48.15 \\
4.5 & 0.0211 & 50.39 & 47.50 \\
5.0 & 0.0200 & 52.88 & 50.01 \\
5.5 & 0.0204 & 51.94 & 49.03 \\
6.0 & 0.0211 & 50.36 & 47.47 \\
6.5 & 0.0190 & 55.18 & 52.57 \\
7.0 & 0.0193 & 54.45 & 51.73 \\
7.5 & 0.0208 & 51.03 & 48.12 \\
8.0 & 0.0195 & 54.09 & 51.33 \\
8.5 & 0.0201 & 52.53 & 49.64 \\
\hline
\end{tabular}

\section{Arrhenius plot for $2 \mathrm{f}$}

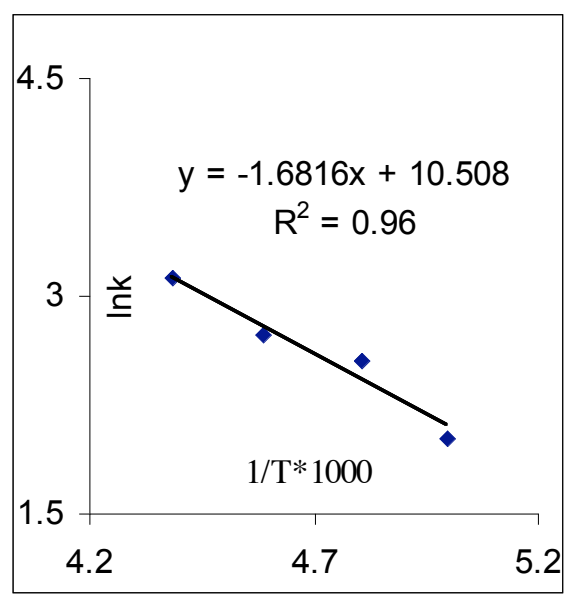

Activation parameters were calculated as before at $200 \mathrm{~K}$, and the results are summarized below.

$\mathrm{Ea}=3.3 \mathrm{kcal} / \mathrm{mol}$

$\mathrm{A}=33900 \mathrm{M}^{-1} \mathrm{~S}^{-1}$

$\Delta \mathrm{H}^{\ddagger}=2.9 \mathrm{kcal} / \mathrm{mol}$

$\Delta \mathrm{S}^{\ddagger}=-39 \mathrm{eu}$

$\Delta \mathrm{G}^{\ddagger}=10.7 \mathrm{kcal} / \mathrm{mol}$ 


\section{References}

(1) Denmark, S. E.; Bui, T. Proc, Nat. Acad. Sci. 2004, 101, 5439-5444.

(2) Denmark, S. E.; Wynn T. J. Am Chem. Soc. 2001, 123, 6199-6200.

(3) (a) Singleton, D. A.; Thomas, A. A. J. Am. Chem. Soc. 1995, 117, 9357-9358. (b)

Singleton, D. A.; Snyder, J. P.; Frantz, D. E.; J. Am. Chem. Soc. 1997, 119, 3383-3384.

(4) Streitwieser, Jr. A. Chem. Rev. 1956, 56, 571-752. 